TRANSACTIONS OF THE

AMERICAN MATHEMATICAL SOCIETY

Volume 352, Number 10, Pages 4843-4875

S 0002-9947(00)02575-7

Article electronically published on June 8, 2000

\title{
A REDUCED TITS QUADRATIC FORM AND TAMENESS OF THREE-PARTITE SUBAMALGAMS OF TILED ORDERS
}

\author{
DANIEL SIMSON
}

Dedicated to Klaus Roggenkamp on the occasion of his 60th birthday

\begin{abstract}
Let $D$ be a complete discrete valuation domain with the unique maximal ideal $\mathfrak{p}$. We suppose that $D$ is an algebra over an algebraically closed field $K$ and $D / \mathfrak{p} \cong K$. Subamalgam $D$-suborders $\Lambda^{\bullet}$ of a tiled $D$-order $\Lambda$ are studied in the paper by means of the integral Tits quadratic form $q_{\Lambda} \bullet$ : $\mathbb{Z}^{n_{1}+2 n_{3}+2} \longrightarrow \mathbb{Z}$. A criterion for a subamalgam $D$-order $\Lambda^{\bullet}$ to be of tame lattice type is given in terms of the Tits quadratic form $q_{\Lambda} \bullet$ and a forbidden list $\Omega_{1}, \ldots, \Omega_{17}$ of minor $D$-suborders of $\Lambda^{\bullet}$ presented in the tables.
\end{abstract}

\section{IntRoduCtion}

Throughout this paper $K$ is an algebraically closed field and $D$ is a complete discrete valuation domain which is a $K$-algebra such that $D / \mathfrak{p} \cong K$, where $\mathfrak{p}$ is the unique maximal ideal of $D$. We denote by $F=D_{0}$ the field of fractions of $D$.

We recall that a $D$-order $\Lambda$ in a finite dimensional semisimple $F$-algebra $C$ is a subring $\Lambda$ of $C$ which is a finitely generated free $D$-submodule of $C$ and $\Lambda$ contains an $F$-basis of $C[5]$. We denote by $\operatorname{latt}(\Lambda)$ the category of right $\Lambda$-lattices, that is, finitely generated right $\Lambda$-modules which are free as $D$-modules. It is well-known that any $D$-order is a semiperfect ring and the category $\operatorname{latt}(\Lambda)$ has the finite unique decomposition property [32, Section 1.1].

A $D$-order $\Lambda$ is said to be of finite lattice type if the category latt $(\Lambda)$ has finitely many isomorphism classes of indecomposable modules. A $D$-order $\Lambda$ is said to be of tame lattice type if the indecomposable $\Lambda$-lattices of any fixed $D$-rank form a finite set of at most one-parameter families (see [9], 34, Section 3], [39. Section 7]). The definitions are presented at the end of this section.

It was shown by the author in [40] that the weak positivity of the reduced Tits quadratic form (1.4) associated with the subamalgam $D$-order $\Lambda^{\bullet}(1.3)$ of tiled $D$-order $\Lambda$ (1.1) is a necessary and sufficient condition for finite lattice type.

Our main result of this paper is the characterization given in Theorem 1.5 below of $D$-orders $\Lambda^{\bullet}(1.3)$ of tame lattice type in terms of the associated Tits quadratic form (1.4) defined below, and by presenting in Section 1A a list of minimal forbidden minor $D$-suborders of $\Lambda^{\bullet}$.

We shall use here the terminology and notation introduced in [40. We denote by $\mathbb{M}_{m}(D)$ the full $m \times m$ matrix ring with coefficients in $D$. We suppose that

Received by the editors November 12, 1997.

2000 Mathematics Subject Classification. Primary 16G30, 16G50, 15A21; Secondary 15A63, 16G60.

Partially supported by Polish KBN Grant 2 P0 3A 01216. 
$n, n_{1}, n_{2}>0$ and $n_{3} \geq 0$ are natural numbers and $\Lambda$ is a tiled $D$-suborder of $\mathbb{M}_{n}(D)$ of the form

$$
\left.\Lambda=\left(\begin{array}{cccc}
D & { }_{1} D_{2} & \ldots & { }_{1} D_{n} \\
\mathfrak{p} & D & \ldots & { }_{2} D_{n} \\
\vdots & \vdots & \ddots & \vdots \\
\mathfrak{p} & \mathfrak{p} & \ldots & { }_{n-1} D_{n} \\
\mathfrak{p} & \mathfrak{p} & \ldots & D
\end{array}\right)\right\} n
$$

such that

(a) ${ }_{i} D_{j}$ is either $D$ or $\mathfrak{p}$, and

(b) $\Lambda$ admits a three-partition

$$
\Lambda=\left(\begin{array}{c|c|c}
\Lambda_{1} & \mathcal{X} & \mathbb{M}_{n_{1}}(D) \\
\hline \mathbb{M}_{n_{3} \times n_{1}}(\mathfrak{p}) & \Lambda_{3} & \mathcal{Y} \\
\hline \mathbb{M}_{n_{1}}(\mathfrak{p}) & \mathbb{M}_{n_{1} \times n_{3}}(\mathfrak{p}) & \Lambda_{2}
\end{array}\right) \begin{gathered}
\} n_{1} \\
\} n_{3} \\
\} n_{2}
\end{gathered}
$$

where $\Lambda_{2}=\Lambda_{1}, n_{1}=n_{2}, n_{1}+n_{2}+n_{3}=n$ and $\Lambda_{3}$ is a hereditary $n_{3} \times n_{3}$ matrix $D$-order

$$
\left.\Lambda_{3}=\left(\begin{array}{ccccc}
D & D & \ldots & D & D \\
\mathfrak{p} & D & \ldots & D & D \\
\vdots & \vdots & \ddots & \vdots & \vdots \\
\mathfrak{p} & \mathfrak{p} & \ldots & D & D \\
\mathfrak{p} & \mathfrak{p} & \ldots & \mathfrak{p} & D
\end{array}\right)\right\} n_{3}
$$

In particular, ${ }_{i} D_{j}=D$ holds in $\Lambda$ for $1 \leq i \leq n_{1}$ and $n_{1}+n_{3}+1 \leq j \leq n$.

Note that $1=\varepsilon_{1}+\varepsilon_{3}+\varepsilon_{2}$, where $\varepsilon_{1}, \varepsilon_{3}$ and $\varepsilon_{2}$ are the matrix idempotents of $\Lambda$ corresponding to the identity elements of $\Lambda_{1}, \Lambda_{3}$ and $\Lambda_{2}$, respectively. By a three-partite subamalgam of $\Lambda$ we shall mean the $D$-suborder

$$
\Lambda^{\bullet}=\left\{\lambda=\left[\lambda_{i j}\right] ; \quad \varepsilon_{1} \lambda \varepsilon_{1}-\varepsilon_{2} \lambda \varepsilon_{2} \in \mathbb{M}_{n_{1}}(\mathfrak{p})\right\}
$$

of $\Lambda$ consisting of all matrices $\lambda=\left[\lambda_{i j}\right]$ of $\Lambda$ such that the left upper corner $n_{1} \times n_{1}$ submatrix $\varepsilon_{1} \lambda \varepsilon_{1}$ of $\lambda$ is congruent modulo $\mathbb{M}_{n_{1}}(\mathfrak{p})$ to the right lower corner $n_{1} \times n_{1}$ submatrix $\varepsilon_{2} \lambda \varepsilon_{2}$ of $\lambda$.

To any such $D$-order $\Lambda^{\bullet}$ we have associated in [40] the reduced Tits quadratic form

$$
q_{\Lambda} \cdot: \mathbb{Z}^{n_{1}+2 n_{3}+2} \longrightarrow \mathbb{Z}
$$

in the indeterminates $x_{*}, x_{+}, x_{1}, \ldots, x_{n_{1}+n_{3}}, \bar{x}_{n_{1}+1}, \ldots, \bar{x}_{n_{1}+n_{3}}$ defined by the formula

$$
\begin{aligned}
q_{\Lambda} \bullet\left(x_{1}, \ldots, x_{n_{1}+n_{3}}, \bar{x}_{n_{1}+1}, \ldots, \bar{x}_{n_{1}+n_{3}}, x_{*}, x_{+}\right) \\
=x_{*}^{2}+x_{+}^{2}+\sum_{j=1}^{n_{1}+n_{3}} x_{j}^{2}+\sum_{j=n_{1}+1}^{n_{1}+n_{3}} \bar{x}_{j}^{2} \\
+\sum_{\substack{i \leq i<j \leq n_{1}+n_{3} \\
1 \leq D_{j}}} x_{i} x_{j}+\sum_{s<t} \bar{x}_{s} \bar{x}_{t}+\sum_{\substack{t D_{s}=D \\
n_{1}<t \leq n_{1}+n_{3}<s}} x_{s-n_{1}-n_{3}} \bar{x}_{t} \\
-x_{+}\left(\sum_{j=1}^{n_{1}+n_{3}} x_{j}\right)-x_{*}\left(\sum_{j=1}^{n_{1}} x_{j}+\sum_{j=n_{1}+1}^{n_{1}+n_{3}} \bar{x}_{j}\right) .
\end{aligned}
$$


Our main result of this paper is the following theorem.

Theorem 1.5. Let $K$ be an algebraically closed field and $D$ a complete discrete valuation domain which is a $K$-algebra such that $D / \mathfrak{p} \cong K$, where $\mathfrak{p}$ is the unique maximal ideal of $D$.

Let $\Lambda$ be a three-partite $D$-order of the form (1.2) and let $\Lambda^{\bullet}$ be the subamalgam (1.3) of $\Lambda \subseteq \mathbb{M}_{n}(D)$, where $\Lambda_{1}=\Lambda_{2} \subseteq \mathbb{M}_{n_{1}}(D), \Lambda_{3} \subseteq \mathbb{M}_{n_{3}}(D)$ and $n_{1}, n_{3}$ are as above. If the part $\mathcal{X}$ or the part $\mathcal{Y}$ of the D-order $\Lambda$ in (1.2) consists of matrices with coefficients in $\mathfrak{p}$, then the following conditions are equivalent.

(a) The D-order $\Lambda^{\bullet}$ is of tame lattice type.

(b) The integral reduced Tits quadratic form $q_{\Lambda} \bullet: \mathbb{Z}^{n_{1}+2 n_{3}+2} \longrightarrow \mathbb{Z}$ (1.4) is weakly non-negative, that is, $q_{\Lambda} \bullet(z) \geq 0$ for any vector $z \in \mathbb{N}^{n_{1}+2 n_{3}+2}$.

(c) Either $n_{3}=0$ and the D-order $\Lambda_{1}$ in (1.2) does not contain minor Dsuborders of one of the forms

$$
\begin{array}{rlrl}
\Delta_{0} & =\left(\begin{array}{lll}
D & \mathfrak{p} & \mathfrak{p} \\
\mathfrak{p} & D & \mathfrak{p} \\
\mathfrak{p} & \mathfrak{p} & D
\end{array}\right), & \Delta_{1}=\left(\begin{array}{lll}
D & \mathfrak{p} & D \\
\mathfrak{p} & D & \mathfrak{p} \\
\mathfrak{p} & \mathfrak{p} & D
\end{array}\right), \\
\Delta_{2}=\left(\begin{array}{lll}
D & D & \mathfrak{p} \\
\mathfrak{p} & D & \mathfrak{p} \\
\mathfrak{p} & \mathfrak{p} & D
\end{array}\right), & \Delta_{3}=\left(\begin{array}{lll}
D & \mathfrak{p} & \mathfrak{p} \\
\mathfrak{p} & D & D \\
\mathfrak{p} & \mathfrak{p} & D
\end{array}\right),
\end{array}
$$

or else $n_{3} \geq 1, \Lambda_{1}$ is hereditary of the form

$$
\left(\begin{array}{ccccc}
D & D & \ldots & D & D \\
\mathfrak{p} & D & \ldots & D & D \\
\vdots & \vdots & \ddots & \vdots & \vdots \\
\mathfrak{p} & \mathfrak{p} & \ldots & D & D \\
\mathfrak{p} & \mathfrak{p} & \ldots & \mathfrak{p} & D
\end{array}\right)
$$

and the three-partite subamalgam D-orders $\Lambda^{\bullet}$ and $\operatorname{rt}(\Lambda)^{\bullet}(1.7)$ do not contain threepartite minor D-suborders dominated by any of the 17 three-partite subamalgam D-orders listed in the tables of Section $1 \mathrm{~A}$.

(d) The two-peak poset $\left(I_{\Lambda}^{*+}, \mathfrak{Z}_{\Lambda} \bullet\right)$ with zero-relations associated with $\Lambda^{\bullet}$ in $(3.3)$ does not contain as a two-peak subposet with zero-relations any of the 13 forms shown in Figure 1 (the dotted line in $\widehat{\mathcal{F}}_{4}$ means a zero-relation).

We recall from [0] that, given a matrix $\lambda \in \mathbb{M}_{n}(D)$, we define the reflection transpose of $\lambda$ to be the transpose matrix $\operatorname{rt}(\lambda) \in \mathbb{M}_{n}(D)$ of $\lambda$ with respect to the non-main diagonal. Given any $D$-order $\Lambda$, we define the reflection transpose of $\Lambda$ (resp. of $\Lambda^{\bullet}$ ) to be the $D$-orders

$$
\operatorname{rt}(\Lambda)=\{\operatorname{rt}(\lambda) ; \lambda \in \Lambda\} \quad\left(\operatorname{resp} \cdot \operatorname{rt}\left(\Lambda^{\bullet}\right)=\left\{\operatorname{rt}(\lambda) ; \lambda \in \Lambda^{\bullet}\right\}\right)
$$

It is easy to see that $\operatorname{rt}\left(\Lambda^{\bullet}\right)=\operatorname{rt}(\Lambda)^{\bullet}$ and the map $\lambda \mapsto \operatorname{rt}(\lambda)$ defines the $\operatorname{ring}$ anti-isomorphisms $\Lambda \stackrel{\simeq}{\longrightarrow} \operatorname{rt}(\Lambda)$ and $\Lambda^{\bullet} \stackrel{\simeq}{\longrightarrow} \operatorname{rt}\left(\Lambda^{\bullet}\right)$.

If $1 \leq i_{1}<\cdots<i_{s} \leq n_{1}$, we say that the order $\Delta$ is an $\left(i_{1}, \ldots, i_{s}\right)$-minor $D$-suborder of $\Lambda_{1}$ in (1.2) if $\Delta$ is obtained from $\Lambda_{1}$ by omitting the $i_{j}$ th row and the $i_{j}$ th column for $j=1, \ldots, s$.

A three-partite order $\Omega$ is said to be a three-partite minor $D$-suborder of $\Lambda^{\bullet}$ if $\Omega$ is a minor $D$-suborder of $\Lambda^{\bullet}$ obtained by omitting rows and columns simultaneously in parts $\Lambda_{1}$ and $\Lambda_{2}$; that is, we omit any $i$-th row and any $i$-th column 


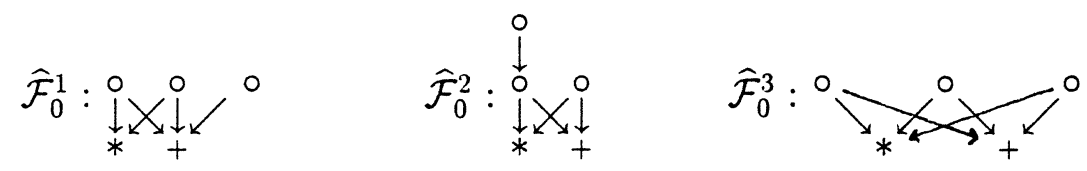

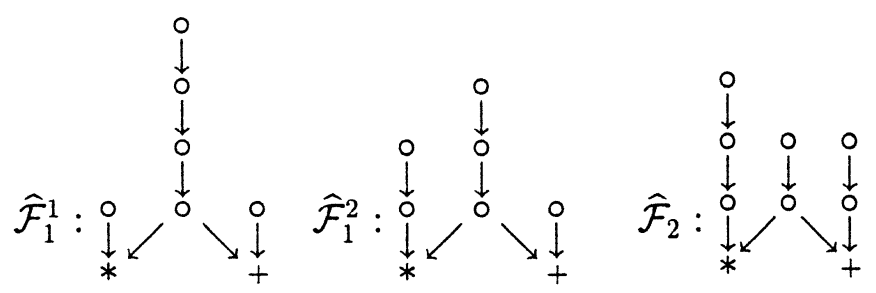
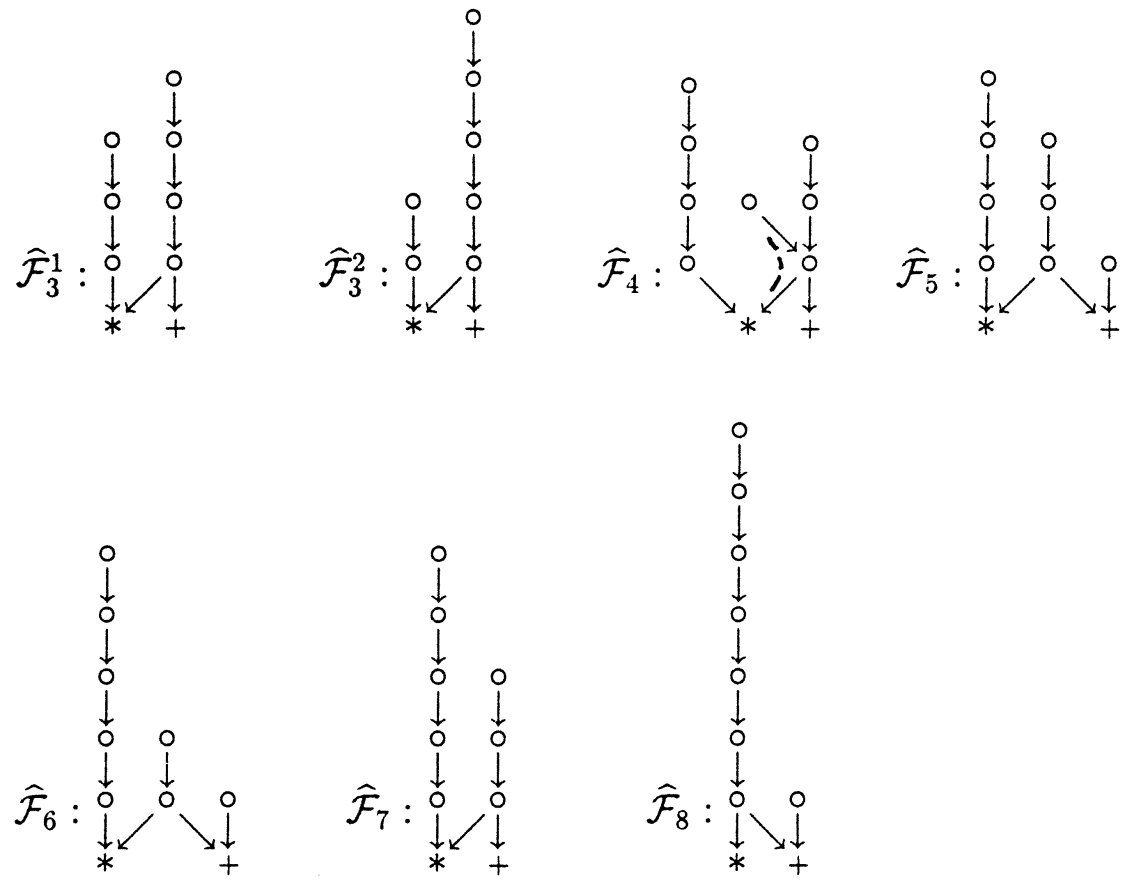

FIGURE 1.

of $\Lambda^{\bullet}$, where $1 \leq i \leq n_{1}$, and simultaneously we omit the $\left(n_{1}+n_{3}+i\right)$-th row and the $\left(n_{1}+n_{3}+i\right)$-th column of $\Lambda^{\bullet}$.

A three-partite subamalgam $D$-order $\Lambda^{\bullet}(1.3)$ is said to be dominated by a three-partite subamalgam $D$-order $\bar{\Lambda}^{\bullet}$ if $\Lambda^{\bullet}$ is a three-partite $D$-suborder of $\bar{\Lambda}^{\bullet}$ of the same size (1.2) and $\Lambda_{1}=\bar{\Lambda}_{1}, \Lambda_{2}=\bar{\Lambda}_{2}, \Lambda_{3}=\bar{\Lambda}_{3}, \mathcal{X} \subseteq \overline{\mathcal{X}}, \mathcal{Y} \subseteq \overline{\mathcal{Y}}$ (see [40], 444] p. 69]).

Let us recall from [9], 32, Section 15.12] and [34, Section 3] the definition of an order of tame lattice type. Let $\Omega$ be an arbitrary $D$-order in a semisimple $D_{0^{-}}$ algebra $C$, where $D$ is a complete discrete valuation domain which is an algebra 
over an algebraically closed field $K$ and $D / \mathfrak{p} \cong K$. Then $\Omega$ is said to be of tame lattice type (or the category $\operatorname{latt}(\Omega)$ is said to be of tame representation type) if for any number $r \in \mathbb{N}$ there exist a non-zero polynomial $h \in K[y]$ and a family of additive functors

$$
(-) \otimes_{A} M^{(1)}, \ldots,(-) \otimes_{A} M^{(s)}: \operatorname{ind}_{1}(A) \longrightarrow \operatorname{latt}(\Omega),
$$

where $A=K\left[y, h^{-1}\right]$, $\operatorname{ind}_{1}(A)$ is the full subcategory of $\bmod (A)$ consisting of one dimensional $A$-modules, and $M^{(1)}, \ldots M^{(s)}$ are $A$ - $\Omega$-bimodules satisfying the following conditions:

(P0) The left $A$-modules ${ }_{A} M^{(1)}, \ldots,{ }_{A} M^{(s)}$ are flat.

(P1) All but finitely many indecomposable $\Omega$-lattices of $D$-rank $r$ are isomorphic to lattices in $\operatorname{Im}(-) \otimes_{A} M^{(1)} \cup \ldots \cup \operatorname{Im}(-) \otimes_{A} M^{(s)}$.

(P2) $M_{\Omega}^{(1)}, \ldots, M_{\Omega}^{(s)}$ viewed as $D$-modules are torsion-free.

(P3) ${ }_{A} M_{\Omega}^{(1)}, \ldots,{ }_{A} M_{\Omega}^{(s)}$ are finitely generated as $A$ - $\Omega$-bimodules.

This means that the functors (1.8) form an almost parameterizing family (see [32.

Definition 14.13]) for the category $\operatorname{ind}_{r}(\operatorname{latt}(\Omega))$ of indecomposable $\Omega$-lattices of $D$-rank $r$.

Given an integer $r \geq 1$, we define $\boldsymbol{\mu}_{\text {latt }(\Omega)}^{1}(r)$ to be the minimal number $s$ of functors (1.8) satisfying the above conditions. The $D$-order $\Omega$ of tame lattice type is defined to be of polynomial growth [34, Section 3] if there exists an integer $g \geq 1$ such that $\boldsymbol{\mu}_{\text {latt }(\Omega)}^{1}(r) \leq r^{g}$ for all integers $r \geq 2($ compare with [32, p. 291]).

It was proved in [9] that the tame-wild dichotomy holds for $D$-orders $\Omega$ under the assumption on $D$ made above. The reader is referred to [9], [34, Section 3], [39. Section 7] for various definitions and discussion of orders of tame lattice type and of wild lattice type.

Our main result, Theorem 1.5, is proved in Section 4 by applying a technique developed in [35] and [40]. In particular, we apply the covering technique for bipartite stratified posets developed by the author in [31], and a reduction functor $\mathbb{H}$ (3.5) from latt $\left(\Lambda^{\bullet}\right)$ to $K$-linear socle projective representations of a two-peak poset $\left(I_{\Lambda^{\bullet}}^{*+}, \mathfrak{Z}_{\Lambda^{\bullet}}\right)(3.3)$ with zero-relations associated with $\Lambda^{\bullet}$ in 40 . Then we apply a criterion for tame prinjective type of two-peak posets given in [17] and [18].

In Section 2 we collect basic facts on $K$-linear socle projective representations of a multi-peak posets with zero-relations we need in this paper.

In Section 3 we associate with $\Lambda^{\bullet}$ a two-peak poset $\left(I_{\Lambda}^{*+}, \mathfrak{Z}_{\Lambda} \bullet\right)$ with zero-relations (see $(3.3)$ ), and we prove in Theorem 3.4 main properties of our reduction functor $\mathbb{H}: \operatorname{latt}\left(\Lambda^{\bullet}\right) \rightarrow\left(I_{\Lambda}^{*+}, \mathfrak{Z}_{\Lambda} \bullet\right)-\operatorname{spr}$.

By applying [40, Theorem 6.1] we get a structure of the Auslander-Reiten quiver $\Gamma\left(\operatorname{latt}\left(\Lambda^{\bullet}\right)\right)$ of $\operatorname{latt}\left(\Lambda^{\bullet}\right)($ see Remark 3.12).

A simple criterion for a tame lattice type $D$-order $\Lambda^{\bullet}(1.3)$ to be of polynomial growth is given by the author in [42, Theorem 1.5]. Tame lattice type subamalgam $D$-orders $\Lambda^{\bullet}(1.3)$ of non-polynomial growth are completely described in [42, Theorem 6.2 and Corollary 6.3].

The main results of this paper were presented at the AMS-IMS-SIAM Joint Summer Research Conference "Trends in the Representation Theory of Finite Dimensional Algebras" at the University of Washington, Seattle, in July 1997 (see [11, Theorem 4.2]). They were also presented at the Euroconference "Interactions between Ring Theory and Representations of Algebras", Murcia, 12-17 January 1998 (see [10] and [43] Section 8]). 


\section{A. Tables of Minimal Three-Partite Subamalgams of Tiled D-orders of Wild Lattice Type}

We use here the notation introduced in Section 1; that is, we present three-partite tiled $D$-orders $\Omega_{j}$, and the three-partition is indicated by vertical and horizontal

lines. The subamalgam $\Omega_{j}^{\bullet}$ is obtained from $\Omega_{j}$ by the identification modulo $\mathfrak{p}=$ $\operatorname{rad}(D)$ the left upper corner block of $\Omega_{j}$ with the right lower corner block of $\Omega_{j}$.

\section{Type $\widehat{\mathcal{F}}_{1}^{1}:$}

$\Omega_{1}=\left(\begin{array}{llll|l|llll}D & D & D & D & \mathfrak{p} & D & D & D & D \\ \mathfrak{p} & D & D & D & \mathfrak{p} & D & D & D & D \\ \mathfrak{p} & \mathfrak{p} & D & D & \mathfrak{p} & D & D & D & D \\ \mathfrak{p} & \mathfrak{p} & \mathfrak{p} & D & \mathfrak{p} & D & D & D & D \\ \hline \mathfrak{p} & \mathfrak{p} & \mathfrak{p} & \mathfrak{p} & D & \mathfrak{p} & \mathfrak{p} & \mathfrak{p} & \mathfrak{p} \\ \hline \mathfrak{p} & \mathfrak{p} & \mathfrak{p} & \mathfrak{p} & \mathfrak{p} & D & D & D & D \\ \mathfrak{p} & \mathfrak{p} & \mathfrak{p} & \mathfrak{p} & \mathfrak{p} & \mathfrak{p} & D & D & D \\ \mathfrak{p} & \mathfrak{p} & \mathfrak{p} & \mathfrak{p} & \mathfrak{p} & \mathfrak{p} & \mathfrak{p} & D & D \\ \mathfrak{p} & \mathfrak{p} & \mathfrak{p} & \mathfrak{p} & \mathfrak{p} & \mathfrak{p} & \mathfrak{p} & \mathfrak{p} & D\end{array}\right) n_{3}=1$

$\Omega_{2}=\left(\begin{array}{llll|ll|llll}D & D & D & D & \mathfrak{p} & D & D & D & D & D \\ \mathfrak{p} & D & D & D & \mathfrak{p} & D & D & D & D & D \\ \mathfrak{p} & \mathfrak{p} & D & D & \mathfrak{p} & D & D & D & D & D \\ \mathfrak{p} & \mathfrak{p} & \mathfrak{p} & D & \mathfrak{p} & D & D & D & D & D \\ \hline \mathfrak{p} & \mathfrak{p} & \mathfrak{p} & \mathfrak{p} & D & D & D & D & D & D \\ \mathfrak{p} & \mathfrak{p} & \mathfrak{p} & \mathfrak{p} & \mathfrak{p} & D & \mathfrak{p} & \mathfrak{p} & \mathfrak{p} & \mathfrak{p} \\ \hline \mathfrak{p} & \mathfrak{p} & \mathfrak{p} & \mathfrak{p} & \mathfrak{p} & \mathfrak{p} & D & D & D & D \\ \mathfrak{p} & \mathfrak{p} & \mathfrak{p} & \mathfrak{p} & \mathfrak{p} & \mathfrak{p} & \mathfrak{p} & D & D & D \\ \mathfrak{p} & \mathfrak{p} & \mathfrak{p} & \mathfrak{p} & \mathfrak{p} & \mathfrak{p} & \mathfrak{p} & \mathfrak{p} & D & D \\ \mathfrak{p} & \mathfrak{p} & \mathfrak{p} & \mathfrak{p} & \mathfrak{p} & \mathfrak{p} & \mathfrak{p} & \mathfrak{p} & \mathfrak{p} & D\end{array}\right) n_{3}=2$,

Type $\widehat{\mathcal{F}}_{1}^{2}$ :

$\Omega_{3}=\left(\begin{array}{lll|ll|lll}D & D & D & \mathfrak{p} & D & D & D & D \\ \mathfrak{p} & D & D & \mathfrak{p} & D & D & D & D \\ \mathfrak{p} & \mathfrak{p} & D & \mathfrak{p} & D & D & D & D \\ \hline \mathfrak{p} & \mathfrak{p} & \mathfrak{p} & D & D & \mathfrak{p} & \mathfrak{p} & \mathfrak{p} \\ \mathfrak{p} & \mathfrak{p} & \mathfrak{p} & \mathfrak{p} & D & \mathfrak{p} & \mathfrak{p} & \mathfrak{p} \\ \hline \mathfrak{p} & \mathfrak{p} & \mathfrak{p} & \mathfrak{p} & \mathfrak{p} & D & D & D \\ \mathfrak{p} & \mathfrak{p} & \mathfrak{p} & \mathfrak{p} & \mathfrak{p} & \mathfrak{p} & D & D \\ \mathfrak{p} & \mathfrak{p} & \mathfrak{p} & \mathfrak{p} & \mathfrak{p} & \mathfrak{p} & \mathfrak{p} & D\end{array}\right) n_{3}=2$,

$\Omega_{4}=\left(\begin{array}{lll|lll|lll}D & D & D & \mathfrak{p} & D & D & D & D & D \\ \mathfrak{p} & D & D & \mathfrak{p} & D & D & D & D & D \\ \mathfrak{p} & \mathfrak{p} & D & \mathfrak{p} & D & D & D & D & D \\ \hline \mathfrak{p} & \mathfrak{p} & \mathfrak{p} & D & D & D & D & D & D \\ \mathfrak{p} & \mathfrak{p} & \mathfrak{p} & \mathfrak{p} & D & D & \mathfrak{p} & \mathfrak{p} & \mathfrak{p} \\ \mathfrak{p} & \mathfrak{p} & \mathfrak{p} & \mathfrak{p} & \mathfrak{p} & D & \mathfrak{p} & \mathfrak{p} & \mathfrak{p} \\ \hline \mathfrak{p} & \mathfrak{p} & \mathfrak{p} & \mathfrak{p} & \mathfrak{p} & \mathfrak{p} & D & D & D \\ \mathfrak{p} & \mathfrak{p} & \mathfrak{p} & \mathfrak{p} & \mathfrak{p} & \mathfrak{p} & \mathfrak{p} & D & D \\ \mathfrak{p} & \mathfrak{p} & \mathfrak{p} & \mathfrak{p} & \mathfrak{p} & \mathfrak{p} & \mathfrak{p} & \mathfrak{p} & D\end{array}\right) n_{3}=3$, 


\section{Type $\widehat{\mathcal{F}}_{2}$ :}

$\Omega_{5}=\left(\begin{array}{ll|lll|ll}D & D & \mathfrak{p} & \mathfrak{p} & D & D & D \\ \mathfrak{p} & D & \mathfrak{p} & \mathfrak{p} & D & D & D \\ \hline \mathfrak{p} & \mathfrak{p} & D & D & D & \mathfrak{p} & \mathfrak{p} \\ \mathfrak{p} & \mathfrak{p} & \mathfrak{p} & D & D & \mathfrak{p} & \mathfrak{p} \\ \mathfrak{p} & \mathfrak{p} & \mathfrak{p} & \mathfrak{p} & D & \mathfrak{p} & \mathfrak{p} \\ \hline \mathfrak{p} & \mathfrak{p} & \mathfrak{p} & \mathfrak{p} & \mathfrak{p} & D & D \\ \mathfrak{p} & \mathfrak{p} & \mathfrak{p} & \mathfrak{p} & \mathfrak{p} & \mathfrak{p} & D\end{array}\right) n_{3}=3$

$\Omega_{6}=\left(\begin{array}{ll|llll|ll}D & D & \mathfrak{p} & \mathfrak{p} & D & D & D & D \\ \mathfrak{p} & D & \mathfrak{p} & \mathfrak{p} & D & D & D & D \\ \hline \mathfrak{p} & \mathfrak{p} & D & D & D & D & D & D \\ \mathfrak{p} & \mathfrak{p} & \mathfrak{p} & D & D & D & \mathfrak{p} & \mathfrak{p} \\ \mathfrak{p} & \mathfrak{p} & \mathfrak{p} & \mathfrak{p} & D & D & \mathfrak{p} & \mathfrak{p} \\ \mathfrak{p} & \mathfrak{p} & \mathfrak{p} & \mathfrak{p} & \mathfrak{p} & D & \mathfrak{p} & \mathfrak{p} \\ \hline \mathfrak{p} & \mathfrak{p} & \mathfrak{p} & \mathfrak{p} & \mathfrak{p} & \mathfrak{p} & D & D \\ \mathfrak{p} & \mathfrak{p} & \mathfrak{p} & \mathfrak{p} & \mathfrak{p} & \mathfrak{p} & \mathfrak{p} & D\end{array}\right) n_{3}=4$,

$\Omega_{7}=\left(\begin{array}{ll|lllll|ll}D & D & \mathfrak{p} & \mathfrak{p} & D & D & D & D & D \\ \mathfrak{p} & D & \mathfrak{p} & \mathfrak{p} & D & D & D & D & D \\ \hline \mathfrak{p} & \mathfrak{p} & D & D & D & D & D & D & D \\ \mathfrak{p} & \mathfrak{p} & \mathfrak{p} & D & D & D & D & D & D \\ \mathfrak{p} & \mathfrak{p} & \mathfrak{p} & \mathfrak{p} & D & D & D & \mathfrak{p} & \mathfrak{p} \\ \mathfrak{p} & \mathfrak{p} & \mathfrak{p} & \mathfrak{p} & \mathfrak{p} & D & D & \mathfrak{p} & \mathfrak{p} \\ \mathfrak{p} & \mathfrak{p} & \mathfrak{p} & \mathfrak{p} & \mathfrak{p} & \mathfrak{p} & D & \mathfrak{p} & \mathfrak{p} \\ \hline \mathfrak{p} & \mathfrak{p} & \mathfrak{p} & \mathfrak{p} & \mathfrak{p} & \mathfrak{p} & \mathfrak{p} & D & D \\ \mathfrak{p} & \mathfrak{p} & \mathfrak{p} & \mathfrak{p} & \mathfrak{p} & \mathfrak{p} & \mathfrak{p} & \mathfrak{p} & D\end{array}\right) n_{3}=5$,

Type $\hat{\mathcal{F}}_{3}^{1}$ :

$\Omega_{8}=\left(\begin{array}{llll|lll|llll}D & D & D & D & D & D & D & D & D & D & D \\ \mathfrak{p} & D & D & D & D & D & D & D & D & D & D \\ \mathfrak{p} & \mathfrak{p} & D & D & D & D & D & D & D & D & D \\ \mathfrak{p} & \mathfrak{p} & \mathfrak{p} & D & D & D & D & D & D & D & D \\ \hline \mathfrak{p} & \mathfrak{p} & \mathfrak{p} & \mathfrak{p} & D & D & D & \mathfrak{p} & \mathfrak{p} & \mathfrak{p} & \mathfrak{p} \\ \mathfrak{p} & \mathfrak{p} & \mathfrak{p} & \mathfrak{p} & \mathfrak{p} & D & D & \mathfrak{p} & \mathfrak{p} & \mathfrak{p} & \mathfrak{p} \\ \mathfrak{p} & \mathfrak{p} & \mathfrak{p} & \mathfrak{p} & \mathfrak{p} & \mathfrak{p} & D & \mathfrak{p} & \mathfrak{p} & \mathfrak{p} & \mathfrak{p} \\ \hline \mathfrak{p} & \mathfrak{p} & \mathfrak{p} & \mathfrak{p} & \mathfrak{p} & \mathfrak{p} & \mathfrak{p} & D & D & D & D \\ \mathfrak{p} & \mathfrak{p} & \mathfrak{p} & \mathfrak{p} & \mathfrak{p} & \mathfrak{p} & \mathfrak{p} & \mathfrak{p} & D & D & D \\ \mathfrak{p} & \mathfrak{p} & \mathfrak{p} & \mathfrak{p} & \mathfrak{p} & \mathfrak{p} & \mathfrak{p} & \mathfrak{p} & \mathfrak{p} & D & D \\ \mathfrak{p} & \mathfrak{p} & \mathfrak{p} & \mathfrak{p} & \mathfrak{p} & \mathfrak{p} & \mathfrak{p} & \mathfrak{p} & \mathfrak{p} & \mathfrak{p} & D\end{array}\right) n_{3}=3$

Type $\widehat{\mathcal{F}}_{3}^{2}$ :

$\Omega_{9}=\left(\begin{array}{lllll|ll|lllll}D & D & D & D & D & D & D & D & D & D & D & D \\ \mathfrak{p} & D & D & D & D & D & D & D & D & D & D & D \\ \mathfrak{p} & \mathfrak{p} & D & D & D & D & D & D & D & D & D & D \\ \mathfrak{p} & \mathfrak{p} & \mathfrak{p} & D & D & D & D & D & D & D & D & D \\ \mathfrak{p} & \mathfrak{p} & \mathfrak{p} & \mathfrak{p} & D & D & D & D & D & D & D & D \\ \hline \mathfrak{p} & \mathfrak{p} & \mathfrak{p} & \mathfrak{p} & \mathfrak{p} & D & D & \mathfrak{p} & \mathfrak{p} & \mathfrak{p} & \mathfrak{p} & \mathfrak{p} \\ \mathfrak{p} & \mathfrak{p} & \mathfrak{p} & \mathfrak{p} & \mathfrak{p} & \mathfrak{p} & D & \mathfrak{p} & \mathfrak{p} & \mathfrak{p} & \mathfrak{p} & \mathfrak{p} \\ \hline \mathfrak{p} & \mathfrak{p} & \mathfrak{p} & \mathfrak{p} & \mathfrak{p} & \mathfrak{p} & \mathfrak{p} & D & D & D & D & D \\ \mathfrak{p} & \mathfrak{p} & \mathfrak{p} & \mathfrak{p} & \mathfrak{p} & \mathfrak{p} & \mathfrak{p} & \mathfrak{p} & D & D & D & D \\ \mathfrak{p} & \mathfrak{p} & \mathfrak{p} & \mathfrak{p} & \mathfrak{p} & \mathfrak{p} & \mathfrak{p} & \mathfrak{p} & \mathfrak{p} & D & D & D \\ \mathfrak{p} & \mathfrak{p} & \mathfrak{p} & \mathfrak{p} & \mathfrak{p} & \mathfrak{p} & \mathfrak{p} & \mathfrak{p} & \mathfrak{p} & \mathfrak{p} & D & D \\ \mathfrak{p} & \mathfrak{p} & \mathfrak{p} & \mathfrak{p} & \mathfrak{p} & \mathfrak{p} & \mathfrak{p} & \mathfrak{p} & \mathfrak{p} & \mathfrak{p} & \mathfrak{p} & D\end{array}\right) n_{3}=2$ 
Type $\hat{\mathcal{F}}_{4}$ :

$\Omega_{10}=\left(\begin{array}{lll|llll|lll}D & D & D & \mathfrak{p} & \mathfrak{p} & \mathfrak{p} & \mathfrak{p} & D & D & D \\ \mathfrak{p} & D & D & \mathfrak{p} & \mathfrak{p} & \mathfrak{p} & \mathfrak{p} & D & D & D \\ \mathfrak{p} & \mathfrak{p} & D & \mathfrak{p} & \mathfrak{p} & \mathfrak{p} & \mathfrak{p} & D & D & D \\ \hline \mathfrak{p} & \mathfrak{p} & D & D & D & D & D & D & D & D \\ \mathfrak{p} & \mathfrak{p} & \mathfrak{p} & \mathfrak{p} & D & D & D & D & D & D \\ \mathfrak{p} & \mathfrak{p} & \mathfrak{p} & \mathfrak{p} & \mathfrak{p} & D & D & D & D & D \\ \mathfrak{p} & \mathfrak{p} & \mathfrak{p} & \mathfrak{p} & \mathfrak{p} & \mathfrak{p} & D & \mathfrak{p} & \mathfrak{p} & D \\ \hline \mathfrak{p} & \mathfrak{p} & \mathfrak{p} & \mathfrak{p} & \mathfrak{p} & \mathfrak{p} & \mathfrak{p} & D & D & D \\ \mathfrak{p} & \mathfrak{p} & \mathfrak{p} & \mathfrak{p} & \mathfrak{p} & \mathfrak{p} & \mathfrak{p} & \mathfrak{p} & D & D \\ \mathfrak{p} & \mathfrak{p} & \mathfrak{p} & \mathfrak{p} & \mathfrak{p} & \mathfrak{p} & \mathfrak{p} & \mathfrak{p} & \mathfrak{p} & D\end{array}\right) n_{3}=4$,

$\Omega_{11}=\left(\begin{array}{lll|lllll|lll}D & D & D & \mathfrak{p} & \mathfrak{p} & \mathfrak{p} & \mathfrak{p} & D & D & D & D \\ \mathfrak{p} & D & D & \mathfrak{p} & \mathfrak{p} & \mathfrak{p} & \mathfrak{p} & D & D & D & D \\ \mathfrak{p} & \mathfrak{p} & D & \mathfrak{p} & \mathfrak{p} & \mathfrak{p} & \mathfrak{p} & D & D & D & D \\ \hline \mathfrak{p} & \mathfrak{p} & \mathfrak{p} & D & D & D & D & D & D & D & D \\ \mathfrak{p} & \mathfrak{p} & \mathfrak{p} & \mathfrak{p} & D & D & D & D & D & D & D \\ \mathfrak{p} & \mathfrak{p} & \mathfrak{p} & \mathfrak{p} & \mathfrak{p} & D & D & D & D & D & D \\ \mathfrak{p} & \mathfrak{p} & \mathfrak{p} & \mathfrak{p} & \mathfrak{p} & \mathfrak{p} & D & D & D & D & D \\ \mathfrak{p} & \mathfrak{p} & \mathfrak{p} & \mathfrak{p} & \mathfrak{p} & \mathfrak{p} & \mathfrak{p} & D & \mathfrak{p} & \mathfrak{p} & D \\ \mathfrak{p} & \mathfrak{p} & \mathfrak{p} & \mathfrak{p} & \mathfrak{p} & \mathfrak{p} & \mathfrak{p} & \mathfrak{p} & D & D & D \\ \hline \mathfrak{p} & \mathfrak{p} & \mathfrak{p} & \mathfrak{p} & \mathfrak{p} & \mathfrak{p} & \mathfrak{p} & \mathfrak{p} & \mathfrak{p} & D & D \\ \mathfrak{p} & \mathfrak{p} & \mathfrak{p} & \mathfrak{p} & \mathfrak{p} & \mathfrak{p} & \mathfrak{p} & \mathfrak{p} & \mathfrak{p} & \mathfrak{p} & D\end{array}\right)$

Type $\hat{\mathcal{F}}_{5}$ :

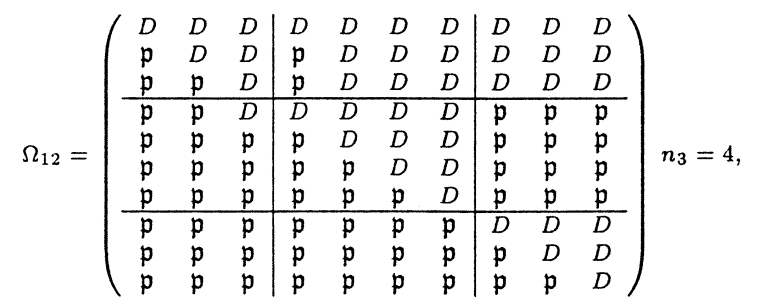

$\Omega_{13}=\left(\begin{array}{lll|lllll|lll}D & D & D & D & D & D & D & D & D & D & D \\ \mathfrak{p} & D & D & \mathfrak{p} & D & D & D & D & D & D & D \\ \mathfrak{p} & \mathfrak{p} & D & \mathfrak{p} & D & D & D & D & D & D & D \\ \mathfrak{p} & \mathfrak{p} & \mathfrak{p} & D & D & D & D & D & D & D & D \\ \hline \mathfrak{p} & \mathfrak{p} & \mathfrak{p} & \mathfrak{p} & D & D & D & D & \mathfrak{p} & \mathfrak{p} & \mathfrak{p} \\ \mathfrak{p} & \mathfrak{p} & \mathfrak{p} & \mathfrak{p} & \mathfrak{p} & D & D & D & \mathfrak{p} & \mathfrak{p} & \mathfrak{p} \\ \mathfrak{p} & \mathfrak{p} & \mathfrak{p} & \mathfrak{p} & \mathfrak{p} & \mathfrak{p} & D & D & \mathfrak{p} & \mathfrak{p} & \mathfrak{p} \\ \mathfrak{p} & \mathfrak{p} & \mathfrak{p} & \mathfrak{p} & \mathfrak{p} & \mathfrak{p} & \mathfrak{p} & D & \mathfrak{p} & \mathfrak{p} & \mathfrak{p} \\ \mathfrak{p} & \mathfrak{p} & \mathfrak{p} & \mathfrak{p} & \mathfrak{p} & \mathfrak{p} & \mathfrak{p} & \mathfrak{p} & D & D & D \\ \hline \mathfrak{p} & \mathfrak{p} & \mathfrak{p} & \mathfrak{p} & \mathfrak{p} & \mathfrak{p} & \mathfrak{p} & \mathfrak{p} & \mathfrak{p} & D & D \\ \mathfrak{p} & \mathfrak{p} & \mathfrak{p} & \mathfrak{p} & \mathfrak{p} & \mathfrak{p} & \mathfrak{p} & \mathfrak{p} & \mathfrak{p} & \mathfrak{p} & D\end{array}\right)$ 
Type $\hat{\mathcal{F}}_{6}:$

$\Omega_{14}=\left(\begin{array}{ll|lllll|ll}D & D & \mathfrak{p} & D & D & D & D & D & D \\ \mathfrak{p} & D & \mathfrak{p} & D & D & D & D & D & D \\ \hline \mathfrak{p} & \mathfrak{p} & D & D & D & D & D & \mathfrak{p} & \mathfrak{p} \\ \mathfrak{p} & \mathfrak{p} & \mathfrak{p} & D & D & D & D & \mathfrak{p} & \mathfrak{p} \\ \mathfrak{p} & \mathfrak{p} & \mathfrak{p} & \mathfrak{p} & D & D & D & \mathfrak{p} & \mathfrak{p} \\ \mathfrak{p} & \mathfrak{p} & \mathfrak{p} & \mathfrak{p} & \mathfrak{p} & D & D & \mathfrak{p} & \mathfrak{p} \\ \mathfrak{p} & \mathfrak{p} & \mathfrak{p} & \mathfrak{p} & \mathfrak{p} & \mathfrak{p} & D & \mathfrak{p} & \mathfrak{p} \\ \hline \mathfrak{p} & \mathfrak{p} & \mathfrak{p} & \mathfrak{p} & \mathfrak{p} & \mathfrak{p} & \mathfrak{p} & D & D \\ \mathfrak{p} & \mathfrak{p} & \mathfrak{p} & \mathfrak{p} & \mathfrak{p} & \mathfrak{p} & \mathfrak{p} & \mathfrak{p} & D\end{array}\right) n_{3}=5$

$\Omega_{15}=\left(\begin{array}{ll|llllll|ll}D & D & \mathfrak{p} & D & D & D & D & D & D & D \\ \mathfrak{p} & D & \mathfrak{p} & D & D & D & D & D & D & D \\ \hline \mathfrak{p} & \mathfrak{p} & D & D & D & D & D & D & D & D \\ \mathfrak{p} & \mathfrak{p} & \mathfrak{p} & D & D & D & D & D & \mathfrak{p} & \mathfrak{p} \\ \mathfrak{p} & \mathfrak{p} & \mathfrak{p} & \mathfrak{p} & D & D & D & D & \mathfrak{p} & \mathfrak{p} \\ \mathfrak{p} & \mathfrak{p} & \mathfrak{p} & \mathfrak{p} & \mathfrak{p} & D & D & D & \mathfrak{p} & \mathfrak{p} \\ \mathfrak{p} & \mathfrak{p} & \mathfrak{p} & \mathfrak{p} & \mathfrak{p} & \mathfrak{p} & D & D & \mathfrak{p} & \mathfrak{p} \\ \mathfrak{p} & \mathfrak{p} & \mathfrak{p} & \mathfrak{p} & \mathfrak{p} & \mathfrak{p} & \mathfrak{p} & D & \mathfrak{p} & \mathfrak{p} \\ \hline \mathfrak{p} & \mathfrak{p} & \mathfrak{p} & \mathfrak{p} & \mathfrak{p} & \mathfrak{p} & \mathfrak{p} & \mathfrak{p} & D & D \\ \mathfrak{p} & \mathfrak{p} & \mathfrak{p} & \mathfrak{p} & \mathfrak{p} & \mathfrak{p} & \mathfrak{p} & \mathfrak{p} & \mathfrak{p} & D\end{array}\right) n_{3}=6$

Type $\hat{\mathcal{F}}_{7}:$

$\Omega_{16}=\left(\begin{array}{lll|lllll|lll}D & D & D & D & D & D & D & D & D & D & D \\ \mathfrak{p} & D & D & D & D & D & D & D & D & D & D \\ \mathfrak{p} & \mathfrak{p} & D & D & D & D & D & D & D & D & D \\ \hline \mathfrak{p} & \mathfrak{p} & \mathfrak{p} & D & D & D & D & D & \mathfrak{p} & \mathfrak{p} & \mathfrak{p} \\ \mathfrak{p} & \mathfrak{p} & \mathfrak{p} & \mathfrak{p} & D & D & D & D & \mathfrak{p} & \mathfrak{p} & \mathfrak{p} \\ \mathfrak{p} & \mathfrak{p} & \mathfrak{p} & \mathfrak{p} & \mathfrak{p} & D & D & D & \mathfrak{p} & \mathfrak{p} & \mathfrak{p} \\ \mathfrak{p} & \mathfrak{p} & \mathfrak{p} & \mathfrak{p} & \mathfrak{p} & \mathfrak{p} & D & D & \mathfrak{p} & \mathfrak{p} & \mathfrak{p} \\ \mathfrak{p} & \mathfrak{p} & \mathfrak{p} & \mathfrak{p} & \mathfrak{p} & \mathfrak{p} & \mathfrak{p} & D & \mathfrak{p} & \mathfrak{p} & \mathfrak{p} \\ \hline \mathfrak{p} & \mathfrak{p} & \mathfrak{p} & \mathfrak{p} & \mathfrak{p} & \mathfrak{p} & \mathfrak{p} & \mathfrak{p} & D & D & D \\ \mathfrak{p} & \mathfrak{p} & \mathfrak{p} & \mathfrak{p} & \mathfrak{p} & \mathfrak{p} & \mathfrak{p} & \mathfrak{p} & \mathfrak{p} & D & D \\ \mathfrak{p} & \mathfrak{p} & \mathfrak{p} & \mathfrak{p} & \mathfrak{p} & \mathfrak{p} & \mathfrak{p} & \mathfrak{p} & \mathfrak{p} & \mathfrak{p} & D\end{array}\right) n_{3}=5$

Type $\hat{\mathcal{F}}_{8}:$

$\Omega_{17}=\left(\begin{array}{lllllll|l|lllllll}D & D & D & D & D & D & D & \mathfrak{p} & D & D & D & D & D & D & D \\ \mathfrak{p} & D & D & D & D & D & D & \mathfrak{p} & D & D & D & D & D & D & D \\ \mathfrak{p} & \mathfrak{p} & D & D & D & D & D & \mathfrak{p} & D & D & D & D & D & D & D \\ \mathfrak{p} & \mathfrak{p} & \mathfrak{p} & D & D & D & D & \mathfrak{p} & D & D & D & D & D & D & D \\ \mathfrak{p} & \mathfrak{p} & \mathfrak{p} & \mathfrak{p} & D & D & D & \mathfrak{p} & D & D & D & D & D & D & D \\ \mathfrak{p} & \mathfrak{p} & \mathfrak{p} & \mathfrak{p} & \mathfrak{p} & D & D & \mathfrak{p} & D & D & D & D & D & D & D \\ \mathfrak{p} & \mathfrak{p} & \mathfrak{p} & \mathfrak{p} & \mathfrak{p} & \mathfrak{p} & D & \mathfrak{p} & D & D & D & D & D & D & D \\ \hline \mathfrak{p} & \mathfrak{p} & \mathfrak{p} & \mathfrak{p} & \mathfrak{p} & \mathfrak{p} & \mathfrak{p} & D & D & D & D & D & D & D & D \\ \hline \mathfrak{p} & \mathfrak{p} & \mathfrak{p} & \mathfrak{p} & \mathfrak{p} & \mathfrak{p} & \mathfrak{p} & \mathfrak{p} & D & D & D & D & D & D & D \\ \mathfrak{p} & \mathfrak{p} & \mathfrak{p} & \mathfrak{p} & \mathfrak{p} & \mathfrak{p} & \mathfrak{p} & \mathfrak{p} & \mathfrak{p} & D & D & D & D & D & D \\ \mathfrak{p} & \mathfrak{p} & \mathfrak{p} & \mathfrak{p} & \mathfrak{p} & \mathfrak{p} & \mathfrak{p} & \mathfrak{p} & \mathfrak{p} & \mathfrak{p} & D & D & D & D & D \\ \mathfrak{p} & \mathfrak{p} & \mathfrak{p} & \mathfrak{p} & \mathfrak{p} & \mathfrak{p} & \mathfrak{p} & \mathfrak{p} & \mathfrak{p} & \mathfrak{p} & \mathfrak{p} & D & D & D & D \\ \mathfrak{p} & \mathfrak{p} & \mathfrak{p} & \mathfrak{p} & \mathfrak{p} & \mathfrak{p} & \mathfrak{p} & \mathfrak{p} & \mathfrak{p} & \mathfrak{p} & \mathfrak{p} & \mathfrak{p} & D & D & D \\ \mathfrak{p} & \mathfrak{p} & \mathfrak{p} & \mathfrak{p} & \mathfrak{p} & \mathfrak{p} & \mathfrak{p} & \mathfrak{p} & \mathfrak{p} & \mathfrak{p} & \mathfrak{p} & \mathfrak{p} & \mathfrak{p} & D & D \\ \mathfrak{p} & \mathfrak{p} & \mathfrak{p} & \mathfrak{p} & \mathfrak{p} & \mathfrak{p} & \mathfrak{p} & \mathfrak{p} & \mathfrak{p} & \mathfrak{p} & \mathfrak{p} & \mathfrak{p} & \mathfrak{p} & \mathfrak{p} & D\end{array}\right) n_{3}=1$ 


\section{Filtered Socle Projective Representations of Posets with Zero-Relations}

We recall from [46] and [32] Chapter 13] that the study of tiled orders is reduced to the study of representations of infinite posets having a unique maximal element. A similar idea applies in the study of some categories of Cohen-Macaulay modules and of abelian groups (see [1, [2], 3], [38, 43]).

We shall prove the main theorems of the paper by reducing the problem for lattices over three-partite subamalgams of tiled $D$-orders to a corresponding problem for $K$-linear socle projective representations of two-peak posets (that is, exactly two maximal elements) with zero-relations that was studied in [31] and 40], where $K=D / \mathfrak{p}$. Our reduction extends the reduction given in [35, Section 2] and involves the reduction functors defined in [1] and 27], and the covering technique for bipartite stratified posets developed by the author in 31.

Throughout we shall denote by $(I ; \preceq)$ a finite poset, that is, a finite partially ordered set $(I ; \preceq)$ with the partial order $\preceq$. We shall write $i \prec j$ if $i \preceq j$ and $i \neq j$. For the sake of simplicity we write $I$ instead of $(I, \preceq)$. We denote by max $I$ the set of all maximal elements of $I$ and $I$ will be called an $r$-peak poset if $|\max I|=r$.

Given a poset $I$, we denote by $K I$ the incidence algebra of $I$ [32], that is, the subalgebra of the full matrix algebra $\mathbb{M}_{I}(K)$ consisting of all $I \times I$ square matrices $\lambda=\left[\lambda_{p q}\right]_{p, q \in I}$ such that $\lambda_{p q}=0$ if $p \npreceq q$ in $(I ; \preceq)$.

For $i \preceq j$ we denote by $e_{i j} \in K I$ the matrix having 1 at the $i$-j-th position and zeros elsewhere. Given $j$ in $I$, we denote by $e_{j}=e_{j j}$ the standard primitive idempotent of $K I$ corresponding to $j$.

The algebra $K I$ is basic, and the standard matrix idempotents $e_{i}, i \in I$, form a complete set of primitive orthogonal idempotents of $K I$. Moreover, $K I$ is of finite global dimension and the right socle of $K I$ is isomorphic to a direct sum of copies of the right ideals $e_{p} K I, p \in \max I$, called the right peaks of $K I$ [33].

We shall denote by $\bmod _{\mathrm{sp}}(K I)$ the category of socle projective right $K I$ modules [28], that is, the full subcategory of $\bmod (K I)$ consisting of modules $X$ such that the socle $\operatorname{soc}(X)$ of $X$ is projective and isomorphic to a direct sum of copies of the right ideals $e_{p} K I, p \in \max I . n$

In our definition of a main reduction functor we shall also need a notion of a poset with zero-relations (see [40]), as follows.

Definition 2.1. A zero-relation in a poset $I$ is a pair $\left(i_{0}, j_{0}\right)$ of elements of $I$ such that $i_{0} \prec j_{0}$.

A set of zero-relations in $I$ is a set $\mathfrak{Z}$ satisfying the following two conditions:

(Z1) $\mathfrak{Z}$ consists of zero-relations $\left(i_{0}, j_{0}\right)$ of $I$.

(Z2) If $\left(i_{0}, j_{0}\right) \in \mathfrak{Z}$ and $i_{1} \preceq i_{0} \preceq j_{0} \preceq j_{1}$, then $\left(i_{1}, j_{1}\right) \in \mathfrak{Z}$.

A right multipeak (or precisely $r$-peak) poset with zero-relations is a pair $(I, \mathfrak{Z})$, where $I$ is a poset, $r=|\max I|$, and $\mathfrak{Z}$ is a set of zero-relations satisfying the following condition (see [30, p. 118]):

(Z3) For every $i \in I \backslash \max I$ there exists $p \in \max I$ such that $(i, p) \notin \mathfrak{Z}$.

In case the set $\mathfrak{Z}$ is empty we shall write $I$ instead of $(I, \mathfrak{Z})$.

A right multipeak poset $\left(I^{\prime}, \mathfrak{Z}^{\prime}\right)$ with zero-relations is said to be a peak subposet of $(I, \mathfrak{Z})$ if $I^{\prime}$ is a subposet of $I, \mathfrak{Z}^{\prime}$ is the restriction of $\mathfrak{Z}$ to $I^{\prime}$ and max $I^{\prime}=$ $I^{\prime} \cap(\max I)$. 
Given a right $r$-peak poset $(I, \mathfrak{Z})$ with zero-relations, we define the incidence $K$-algebra of $(I, \mathfrak{Z})$ to be the $K$-algebra

$$
K(I, \mathfrak{Z})=\left\{\lambda=\left[\lambda_{i j}\right]_{i, j \in I} \in K I ; \lambda_{i j}=0, \text { for }(i, j) \in \mathfrak{Z}\right\} \subseteq K I
$$

consisting of all $I \times I$ square matrices $\lambda=\left[\lambda_{i j}\right]_{i, j \in I} \in \mathbb{M}_{I}(K)$ such that $\lambda_{i j}=0$ if $i \npreceq j$ in $(I ; \preceq)$, or if $(i, j) \in \mathfrak{Z}$. The addition in $K(I, \mathfrak{Z})$ is the usual matrix addition, whereas the multiplication of two matrices $\lambda=\left[\lambda_{i j}\right]_{i, j \in I}$ and $\lambda^{\prime}=\left[\lambda_{i j}^{\prime}\right]_{i, j \in I}$ in $K(I, \mathfrak{Z})$ is the matrix $\lambda^{\prime \prime}=\left[\lambda_{i j}^{\prime \prime}\right]_{i, j \in I}$, where

$$
\lambda_{i j}^{\prime \prime}= \begin{cases}\sum_{i \preceq s \preceq j} \lambda_{i s} \lambda_{s j}^{\prime} & \text { if } i \preceq j \text { and }(i, j) \notin \mathfrak{Z}, \\ 0 & \text { if } i \npreceq j \text { or }(i, j) \in \mathfrak{Z} .\end{cases}
$$

In case the set $\mathfrak{Z}$ is empty we get $K I=K(I, \mathfrak{Z})$.

Note that in case the set $\mathfrak{Z}$ is not empty the algebra $K(I, \mathfrak{Z})$ is not a subalgebra of the matrix algebra $K I \subseteq \mathbb{M}_{I}(K)$.

The incidence algebra $K(I, \mathfrak{Z})$ is basic, and the standard matrix idempotents $e_{i}$, $i \in I$, form a complete set of primitive orthogonal idempotents of $K(I, \mathfrak{Z})$. It is easy to see that $K(I, \mathfrak{Z})$ is a factor $K$-algebra of $K I$ modulo the ideal generated by all matrices $e_{i j} \in K I$ such that $(i, j) \in \mathfrak{Z}$. It follows that the global dimension of $K(I, \mathfrak{Z})$ is finite (see [33. Lemma 2.1]) and, in view of (Z3), the right socle of $K(I, \mathfrak{Z})$ is isomorphic to a direct sum of copies of the right ideals $e_{p} K(I, \mathfrak{Z})$, $p \in \max I$, called the right peaks of $K(I, \mathfrak{Z})$ (see [28]).

We shall denote by $\bmod _{\mathrm{sp}} K(I, \mathfrak{Z})$ the category of socle projective right $K(I, \mathfrak{Z})$-modules, that is, the full subcategory of $\bmod K(I, \mathfrak{Z})$ consisting of modules $X$ such that the $\operatorname{socle} \operatorname{soc}(X)$ of $X$ is projective and isomorphic to a direct sum of copies of the right ideals $e_{p} K(I, \mathfrak{Z}), p \in \max I$ (see [28]).

The category $\bmod _{\mathrm{sp}} K(I, \mathfrak{Z})$ is closed under extensions, direct sums and summands in $\bmod K(I, \mathfrak{Z})$, and has Auslander-Reiten sequences, source maps and sink maps, enough relative projective and enough relative injective objects (see [23]).

Throughout we shall denote by $\operatorname{rep}_{K}(I, \mathfrak{Z})$ the category of $K$-linear representation of $(I, \mathfrak{Z})$, that is, the systems

$$
\left(X_{i},{ }_{j} h_{i}\right)_{i, j \in I, i \prec j}
$$

of finite dimensional $K$-vector spaces $X_{j}$ connected by $K$-linear maps ${ }_{j} h_{i}: X_{i} \rightarrow X_{j}$ satisfying the following conditions:

- ${ }_{i} h_{i}$ is the identity map on $X_{i}$ for any $i \in I$,

- ${ }_{j} h_{i}=0$ if $(i, j) \in \mathfrak{Z}$,

- ${ }_{t} h_{j} \cdot{ }_{j} h_{i}={ }_{t} h_{i}$ if $i \preceq j \preceq t$.

It is well known that there exists a $K$-linear equivalence of categories

$$
\bmod K(I, \mathfrak{Z}) \simeq \operatorname{rep}_{K}(I, \mathfrak{Z})
$$

defined as follows. If $X$ is a module in $\bmod K(I, \mathfrak{Z})$, we define the representation $\left(X_{i},{ }_{j} h_{i}\right)_{i, j \in I, i \prec j}$ in $\operatorname{rep}_{K}(I, \mathfrak{Z})$ by setting $X_{i}=X e_{i}$, and we take for ${ }_{j} h_{i}: X_{i} \rightarrow X_{j}$ the $K$-linear map defined by the multiplication by $e_{i j} \in K(I, \mathfrak{Z})$. Conversely, if the system $\left(X_{i},{ }_{j} h_{i}\right)_{i, j \in I, i \prec j}$ in $\operatorname{rep}_{K}(I, \mathfrak{Z})$ is given, we set $X=\bigoplus_{i \in I} X_{i}$ and we define the multiplication $\cdot: X \times K(I, \mathfrak{Z}) \rightarrow X$ by $x_{i} \cdot e_{i j}={ }_{j} h_{i}\left(x_{i}\right)$ for $x_{i} \in X_{i}$ and $i \preceq j,(i, j) \notin \mathfrak{Z}$. Throughout we shall identify the categories $\bmod K(I, \mathfrak{Z})$ and $\operatorname{rep}_{K}(I, \mathfrak{Z})$ along the functor $X \mapsto\left(X_{i},{ }_{j} h_{i}\right)_{i, j \in I, i \prec j}(2.3)$. 
The module $X$ is socle projective if and only if $X$ viewed as a $K$-linear representation $X=\left(X_{i},{ }_{j} h_{i}\right)_{i, j \in I, i \prec j}$ of $(I, \mathfrak{Z})$ is socle projective, that is, if $\bigcap_{p \in \max I}$ Ker ${ }_{p} h_{i}=$ 0 for any $i \in I \backslash \max I$ (see [28]). It is often useful to deal with filtered forms of socle projective $K$-linear representations of $(I, \mathfrak{Z})$. For this purpose we introduced in [40] the following definition (see also [47, 48]).

Definition 2.4. Let $K$ be a field and let $(I, \mathfrak{Z})$ be a right multipeak poset with zerorelations. A peak $(I, \mathfrak{Z}$ )-space (or a filtered socle projective representation of $(I, \mathfrak{Z}))$ over the field $K$ is the system $\mathbf{M}=\left(M_{j}\right)_{j \in I}$ of finite dimensional $K$-vector spaces $M_{j}$ satisfying the following four conditions.

(a) For any $j \in I$ the $K$-space $M_{j}$ is a $K$-subspace of $M^{\bullet}=\bigoplus_{p \in \max I} M_{p}$.

(b) The inclusion $M_{p} \subseteq M^{\bullet}$ is the standard $p$-coordinate embedding for any $p \in \max I$.

(c) $\pi_{j}\left(M_{i}\right) \subseteq M_{j}$ for all $i \prec j$ in $I$, where $\pi_{j}: M^{\bullet} \rightarrow M^{\bullet}$ is the composed $K$-linear endomorphism

$$
M^{\bullet} \stackrel{\pi_{j}^{\prime}}{\longrightarrow} \bigoplus_{j \preceq p \in \max I} M_{p} \hookrightarrow M^{\bullet}
$$

of $M^{\bullet}$ and $\pi_{j}^{\prime}$ is the direct summand projection.

(d) If $p \in \max I$ and either $i \npreceq p$ or $i \prec p$ and $(i, p) \in \mathfrak{Z}$, then $\pi_{p}\left(M_{i}\right)=0$.

A morphism $f: \mathbf{M} \rightarrow \mathbf{M}^{\prime}$ from $\mathbf{M}$ to $\mathbf{M}^{\prime}$ is a system $f=\left(f_{p}\right)_{p \in \max I}$ of $K$-linear maps $f_{p}: M_{p} \rightarrow M_{p}^{\prime}, p \in \max I$, such that $\left(\bigoplus_{p \in \max I} f_{p}\right)\left(M_{j}\right) \subseteq M_{j}^{\prime}$ for all $j \in I$.

We denote by $(I, \mathfrak{Z})$-spr the category of peak $I$-spaces (or filtered socle projective representations of $(I, \mathfrak{Z})$ ) over the field $K$. The direct sum and the indecomposablity in the category $(I, \mathfrak{Z})$-spr are defined in an obvious way.

A sequence $0 \rightarrow \mathbf{M}^{\prime} \rightarrow \mathbf{M} \rightarrow \mathbf{M}^{\prime \prime} \rightarrow 0$ in the category $(I, \mathfrak{Z})$-spr is said to be exact if the sequence $0 \rightarrow M_{j}^{\prime} \rightarrow M_{j} \rightarrow M_{j}^{\prime \prime} \rightarrow 0$ of vector spaces is exact for every $j \in I$.

In case the set $\mathfrak{Z}$ is empty the category $(I, \mathfrak{Z})$-spr is the category $I$-spr of peak $I$-spaces (or socle projective representations of $I$ ) introduced in 33 .

Let us present an alternative definition of peak $(I, \mathfrak{Z})$-spaces. For this purpose we assume that $\mathbf{M}=\left(M_{j}\right)_{j \in I}$ is system of finite dimensional $K$-vector spaces $M_{j}$. We associate with $\mathbf{M}=\left(M_{j}\right)_{j \in I}$ the $K$-linear representation

$$
\mathbf{M}^{\bullet}=\left(M_{j}^{\bullet},{ }_{i} \pi_{j}^{\bullet}\right)_{j \in I, i \preceq j}
$$

of the poset $I$, where

$$
M_{j}^{\bullet}=\bigoplus_{\substack{j \prec p \in \max \\(j, p) \notin \mathfrak{Z}}} M_{p} \subseteq M^{\bullet}=\bigoplus_{j \prec p \in \max I} M_{p}
$$

and if the relation $i \preceq j$ holds in $I$ we define ${ }_{i} \pi_{j}^{\bullet}: M_{i}^{\bullet} \longrightarrow M_{j}^{\bullet}$ to be the composed $K$-linear map

$$
M_{i}^{\bullet} \subseteq M^{\bullet} \stackrel{\pi_{j}^{\prime}}{\longrightarrow} M_{j}^{\bullet}
$$

where $\pi_{j}^{\prime}$ is the direct summand projection.

The following useful fact is easily verified.

Lemma 2.7. Let $K$ be a field and let $(I, \mathfrak{Z})$ be a right multipeak poset with zerorelations. Assume that $\mathbf{M}=\left(M_{j}\right)_{j \in I}$ is a system of finite dimensional $K$-vector spaces $M_{j}$ and $\mathbf{M}^{\bullet}=\left(M_{j}^{\bullet},{ }_{i} \pi_{j}^{\bullet}\right)_{j \in I, i \preceq}$ is the $K$-linear representation associated with $\mathbf{M}$ above. 
(a) If $i \in I$ and $j \preceq t \preceq s$, then ${ }_{i} \pi_{i}^{\bullet}=$ id and ${ }_{s} \pi_{t}^{\bullet} \cdot{ }_{t} \pi_{j}^{\bullet}={ }_{s} \pi_{j}^{\bullet}$. If $(i, j) \in \mathfrak{Z}$ then ${ }_{i} \pi_{j}^{\bullet}=0$.

(b) The system $\mathbf{M}=\left(M_{j}\right)_{j \in I}$ is a peak $(I, \mathfrak{Z})$-space if and only if the following two conditions are satisfied:

(i) $M_{j} \subseteq M_{j}^{\bullet} \subseteq M^{\bullet}$ for all $j \in I$, and

(ii) ${ }_{j} \pi_{i}^{\bullet}\left(M_{i}\right) \subseteq M_{j}$ if $i \preceq j$-that is, there is a unique factorisation ${ }_{j} \pi_{i}: M_{i} \rightarrow$ $M_{j}$ making the diagram

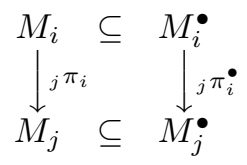

commutative.

It is easy to see that $(I, \mathfrak{Z})$-spr is an additive category with the finite unique decomposition property [32, Section 1.1], and the $K$-linear functor

$$
\rho:(I, \mathfrak{Z})-\operatorname{spr} \simeq \bmod _{\mathrm{sp}} K(I, \mathfrak{Z}),
$$

$\mathbf{M} \mapsto \widehat{\mathbf{M}}=\left(M_{j} ;{ }_{j} \pi_{i}\right)_{i \prec j}$, is an equivalence of categories, where ${ }_{j} \pi_{i}: M_{i} \rightarrow M_{j}$ is the unique $K$-linear map making the diagram (2.8) commutative. The quasi-inverse of $\boldsymbol{\rho}$ is the restriction to the category $\bmod _{\mathrm{sp}} K(I, \mathfrak{Z})$ of the adjustment functor (see [28], 23], [32] (11.32)], [33])

$$
\boldsymbol{\theta}: \bmod K(I, \mathfrak{Z}) \longrightarrow K(I, \mathfrak{Z}) \text {-spr }
$$

associating to $X=\left(X_{i},{ }_{j} h_{i}\right)_{i, j \in I, i \prec j}$ the peak $(I, \mathfrak{Z})$-space $\mathbf{M}(X)=\left(M(X)_{j}\right)_{j \in I}$, where

$$
M(X)_{j}= \begin{cases}X_{j} & \text { for } j \in \max I, \\ \operatorname{Im}\left[\left({ }_{p} h_{j}\right)_{p \in \max I}: X_{j} \rightarrow \bigoplus_{p \in \max I} X_{p}\right] & \text { for } j \in I \backslash \max I .\end{cases}
$$

Corollary 2.11. (a) The category $(I, \mathfrak{Z})$-spr is an additive $K$-category with the finite unique decomposition property [32, Section 1.1].

(b) Every object in $(I, \mathfrak{Z})$-spr has a projective cover.

(c) The category $(I, \mathfrak{Z})$-spr has Auslander-Reiten sequences, source maps and sink maps, enough projective objects and enough relative injective objects [23.

Proof. In view of the equivalence (2.9) the corollary follows by applying the results in 23] to the bipartite algebra $R=K(I, \mathfrak{Z})$ equipped with the bipartition

$$
R=K\left(I_{\Lambda}^{*+}, \mathfrak{Z}_{\Lambda} \bullet\right)=\left(\begin{array}{cc}
A & M \\
0 & B
\end{array}\right)
$$

where

$$
\begin{gathered}
A=K\left(I_{\Lambda}^{*+} \backslash \max I_{\Lambda}^{*+}, \mathfrak{Z}_{\Lambda} \bullet\right) \cong e_{-} R e_{-} \cong R / \operatorname{soc}\left(R_{R}\right), \\
B=K\left(\max I_{\Lambda^{\bullet}}^{*+}\right) \cong e_{+} R e_{+} \cong K \times K \times \cdots \times K \quad\left(\left|\max I_{\Lambda}^{*+}\right|-\text { times }\right), \\
e_{-}=\sum_{j \in I_{\Lambda}^{*+} \backslash \max I_{\Lambda}^{*+}} e_{j}, \quad e_{+}=\sum_{p \in \max I_{\Lambda}^{*+}} e_{p},
\end{gathered}
$$

and the vector space

$$
M=\bigoplus_{p \in \max I_{\Lambda}^{*+} \bullet} \bigoplus_{\substack{j \prec p \\(j, p) \notin \mathfrak{Z}_{\Lambda} \bullet}} e_{i p} K \cong e_{-} R e_{+}
$$


is viewed as an $A$ - $B$-bimodule in an obvious way and multiplication is given by the usual matrix multiplication formula

$$
\left(\begin{array}{cc}
a & m \\
0 & b
\end{array}\right)\left(\begin{array}{cc}
a^{\prime} & m^{\prime} \\
0 & b^{\prime}
\end{array}\right)=\left(\begin{array}{cc}
a a^{\prime} & a m^{\prime}+m b^{\prime} \\
0 & b b^{\prime}
\end{array}\right)
$$

for $a, a^{\prime} \in A, b, b^{\prime} \in B$ and $m, m^{\prime} \in M$.

Note that $(I, \mathfrak{Z})$-spr $\cong \bmod _{\mathrm{sp}} K(I, \mathfrak{Z})$ is the category $\bmod _{i c}(R)$ of injectively cogenerated modules in the notation of [23].

Following [32, Section 14.4], we say that the categories $\bmod _{\mathrm{sp}} K(I, \mathfrak{Z}) \cong$ $(I, \mathfrak{Z})$-spr are of tame representation type if for any number $r \in \mathbb{N}$ there exist a non-zero polynomial $h \in K[y]$ and a family of additive functors

$$
(-) \otimes_{S} N^{(1)}, \ldots,(-) \otimes_{S} N^{(s)}: \operatorname{ind}_{1}(S) \longrightarrow \bmod _{\mathrm{sp}} K(I, \mathfrak{Z}),
$$

where $S=K\left[y, h^{-1}\right], N^{(1)}, \ldots, N^{(s)}$ are $A$ - $K(I, \mathfrak{Z})$-bimodules satisfying the following conditions:

(T0) The left $A$-modules ${ }_{S} N^{(1)}, \ldots,{ }_{S} N^{(s)}$ are finitely generated.

(T1) All but finitely many indecomposable modules in $\bmod _{\mathrm{sp}} K(I, \mathfrak{Z})$ of $K$ dimension $r$ are isomorphic to modules in $\operatorname{Im}(-) \otimes_{S} N^{(1)} \cup \cdots \cup \operatorname{Im}(-) \otimes_{S} N^{(s)}$.

This means that the functors (2.12) form an almost parameterizing family (see [32, Definition 14.13]) for the category $\operatorname{ind}_{r}\left(\bmod _{\mathrm{sp}} K(I, \mathfrak{Z})\right)$ of indecomposable modules $X$ in $\bmod _{\text {sp }} K(I, \mathfrak{Z})$ such that $\operatorname{dim}_{K} X=r$.

Given an integer $r \geq 1$, we define $\boldsymbol{\mu}_{\bmod _{\mathrm{sp}} K(I, \mathfrak{Z})}^{1}(r)$ to be the minimal number $s$ of functors $(2.12)$ satisfying the above conditions. The categories $\bmod _{\mathrm{sp}} K(I, \mathfrak{Z}) \cong$ $(I, \mathfrak{Z})$-spr of tame representation type are defined to be of polynomial growth [34] if there exists an integer $g \geq 1$ such that $\boldsymbol{\mu}_{\bmod _{\mathrm{sp}} K(I, \mathfrak{Z})}^{1}(r) \leq r^{g}$ for all integers $r \geq 2$ (compare with [32, p. 291]).

Following [23] and [33, (3.1)] we associate to any $r$-peak poset $(I, \mathfrak{Z})$ with zerorelations the integral bilinear form $b_{(I, \mathfrak{3})}: \mathbb{Z}^{I} \times \mathbb{Z}^{I} \longrightarrow \mathbb{Z}$,

$$
b_{(I, \mathfrak{Z})}(x, y)=\sum_{j \in I} x_{j} y_{j}+\sum_{\substack{i \prec j \notin \max I \\(i, j) \notin \mathfrak{Z}}} y_{i} x_{j}-\sum_{p \in \max I} \sum_{\substack{i \prec p \\(i, p) \notin \mathfrak{Z}}} x_{i} y_{p} .
$$

and the integral Tits quadratic form $q_{(I, \mathfrak{Z})}: \mathbb{Z}^{I} \longrightarrow \mathbb{Z}, q_{(I, \mathfrak{Z})}(z)=b_{(I, \mathfrak{Z})}(z, z)$.

The following result is useful in applications.

Theorem 2.14. Let $(I, \mathfrak{Z})$ be a multi-peak poset with zero-relations and let $b_{(I, \mathfrak{Z})}$ : $\mathbb{Z}^{I} \times \mathbb{Z}^{I} \rightarrow \mathbb{Z}$ be the bilinear form (2.13).

(a) For any pair $X$ and $Y$ of modules in prin $K(I, \mathfrak{Z})$ (see [40, Section 3]) the following equality holds:

$$
b_{(I, \mathfrak{Z})}(\operatorname{cdn} X, \mathbf{c d n} Y)=\operatorname{dim}_{K} \operatorname{Hom}_{K(I, \mathfrak{Z})}(X, Y)-\operatorname{dim}_{K} \operatorname{Ext}_{K(I, \mathfrak{Z})}^{1}(X, Y)
$$

(b) If the category $(I, \mathfrak{Z})$-spr is of tame representation type then the Tits quadratic form $q_{(I, \mathfrak{Z})}: \mathbb{Z}^{I} \rightarrow \mathbb{Z}$ (see (2.13)) is weakly non-negative.

Proof. The statement (a) follows from [23 Proposition 4.4].

(b) We recall from [41, Proposition 2.7] that there exists an adjustment functor

$$
\boldsymbol{\theta}_{I}: \operatorname{prin} K(I, \mathfrak{Z}) \longrightarrow \bmod _{\mathrm{sp}} K(I, \mathfrak{Z}) \cong(I, \mathfrak{Z}) \text {-spr }
$$


which preserves and reflects tame representation type. Hence the tameness of the category $(I, \mathfrak{Z})$-spr yields the tameness of $\bmod _{\mathrm{sp}} K(I, \mathfrak{Z})$, and consequently the tameness of the category prin $K(I, \mathfrak{Z})$. Then (b) is a consequence of [23, Proposition 4.2] (see also [15] Theorem 3.18]).

We shall often use a reflection duality for the category $(I, \mathfrak{Z})$-spr introduced for socle projective modules in [30]. We shall present it here in a more convenient form.

For this purpose we associate with any $s$-peak poset $(I, \mathfrak{Z})$ with zero-relations, $s \geq 1$, the reflection-dual $s$-peak poset $\left(I^{\bullet}, \mathfrak{Z}^{\bullet}\right)$ with zero-relations defined as follows.

Definition 2.16. Assume that $(I, \mathfrak{Z})$ is an $s$-peak poset with zero-relations, and let $\max I=\left\{p_{1}, \ldots, p_{s}\right\}$.

(a) We define a left-right $s$-peak poset with zero-relations $(\widehat{I}, \widehat{\mathfrak{Z}})$, where

$$
\widehat{I}=\left\{p_{1}^{-}, \ldots, p_{s}^{-}\right\} \cup I
$$

is a poset enlargement of $I$ by a set $\left\{p_{1}^{-}, \ldots, p_{s}^{-}\right\}$of minimal elements. The partial order $\preceq$ in $\widehat{I}$ is an extension of the partial order $\preceq$ in $I$ by the relations

$$
p_{h}^{-} \prec j \Leftrightarrow \text { there exists } i \preceq j \text { in } I \text { such that } i \prec p_{h} \text { in } I \text { and }\left(i, p_{h}\right) \notin \mathfrak{Z}
$$

for any $p_{h} \in \max I$. We define the set $\widehat{\mathfrak{Z}}$ of zero-relations in $\widehat{I}$ to be the set generated by the union of $\mathfrak{Z}$ and the set consisting of the following relations:

- $\left(p_{h}^{-}, p_{t}\right)$ for all $h \neq t$, and

- $\left(p_{h}^{-}, j\right)$, where $p_{h}^{-} \prec j$ holds in $(\widehat{I}, \preceq)$, whereas $j \prec p_{h}$ does not hold in $(I, \preceq)$.

(b) We define the reflection-dual $s$-peak poset with zero-relations

$$
(I, \mathfrak{Z})^{\bullet}=\left(I^{\bullet}, \mathfrak{Z}^{\bullet}\right)
$$

to the poset $(I, \mathfrak{Z})$ to be the poset $I^{\bullet}=(\widehat{I} \backslash \max I)^{\text {op }}$ dual to $(\widehat{I} \backslash \max I, \preceq)$. We take for $\mathfrak{Z}^{\bullet}$ the dual of the restriction of $\widehat{\mathfrak{Z}}$ to $\widehat{I} \backslash \max I$.

Example 2.18. Let

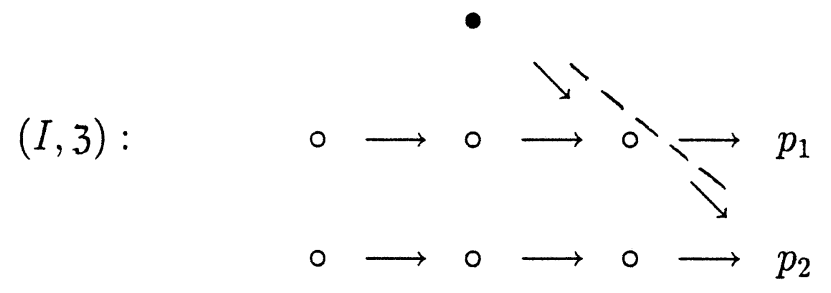

where $\mathfrak{Z}=\left\{\left(\bullet, p_{2}\right)\right\}$ consists of one zero-relation $\left(\bullet, p_{2}\right)$. Then

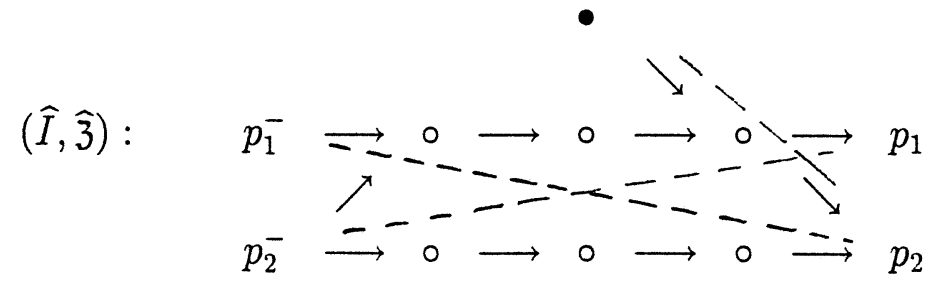

where $\widehat{\mathfrak{Z}}=\left\{\left(\bullet, p_{2}\right),\left(p_{1}^{-}, p_{2}\right),\left(p_{2}^{-}, p_{1}\right)\right\}$ and the dotted lines mean zero-relations. The reflection-dual $\left(I^{\bullet}, \mathfrak{Z}^{\bullet}\right)$ of $(I, \mathfrak{Z})$ is the two-peak poset without zero-relations 


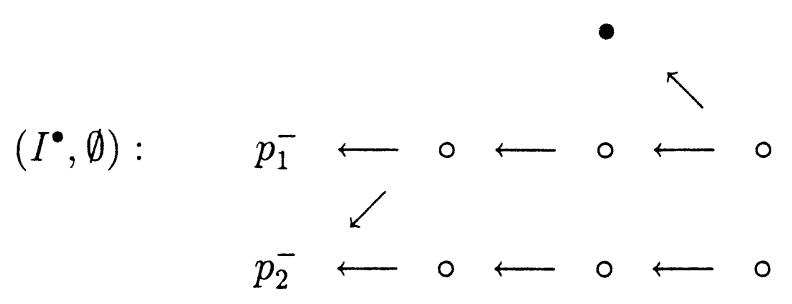

that is, the set $\mathfrak{Z}^{\bullet}$ is empty. Note also that the two-peak poset $\widehat{\mathcal{F}}_{5}$ in Theorem 1.5 (d) is a reflection-dual to $\widehat{\mathcal{F}}_{4}$.

Following [30, 2.6] and [32] Chapter 5], we define a pair of reflection duality functors

$$
(I, \mathfrak{Z})-\operatorname{spr} \underset{D^{\bullet}}{\stackrel{D^{\bullet}}{\leftrightarrows}}(I, \mathfrak{Z})^{\bullet} \text {-spr }
$$

as follows. Given $\mathbf{M}=\left(M_{i}\right)_{i \in I}$ in $(I, \mathfrak{Z})$-spr, we define $D^{\bullet}(\mathbf{M})=\left(\widetilde{M}_{i}\right)_{i \in I} \bullet$, where $\widetilde{M}_{p^{-}}=M_{p}^{*}=\operatorname{Hom}_{K}\left(M_{p}, K\right)$ for $p \in \max I$, and $\widetilde{M}_{j}$ is the image of the $K$-dual vector space to the cokernel $\bar{M}_{j}$ of the embedding

$$
u_{j}: M_{j} \hookrightarrow M_{j}^{\bullet}=\bigoplus_{\substack{j \preceq p \in \max I \\(j, p) \notin \mathfrak{Z}}} M_{p}
$$

under the composed map

$$
\left(\text { Coker } u_{j}\right)^{*} \stackrel{v_{j}^{*}}{\longrightarrow}\left(M_{j}^{\bullet}\right)^{*} \hookrightarrow \widetilde{M} \bullet=\bigoplus_{p^{-} \in \max I} \widetilde{M}_{p^{-}}
$$

and $v_{j}^{*}$ is the $K$-dual map to the cokernel epimorphism $v_{j}: M_{j}^{\bullet} \rightarrow$ Coker $u_{j}$ for $j \in I \backslash \max I$. The functor $D^{\bullet}$ is defined on morphisms in a natural way.

One has to note that $D^{\bullet}(\mathbf{M})$ is an object of $(I, \mathfrak{Z})^{\bullet}$-spr. This easily follows by applying Lemma 2.7 and the following equalities:

$$
M_{j}^{\bullet}=\bigoplus_{\substack{j \preceq p \in \max I \\(j, p) \notin \mathcal{Z}}} M_{p}=\bigoplus_{\substack{j \succeq p^{-} \in \min \widehat{I} \\\left(p^{-}, j\right) \notin \widehat{\mathcal{Z}}}} M_{p}=\left(\widetilde{M}_{j}^{\bullet}\right)^{*}
$$

It follows that the $K$-dual space to $M_{j}^{\bullet}$ is just the space $\widetilde{M}_{j}^{\bullet}$, and the exact sequence

$$
0 \longrightarrow M_{j} \stackrel{u_{j}}{\longrightarrow} M_{j}^{\bullet} \stackrel{v_{j}}{\longrightarrow} \bar{M}_{j} \longrightarrow 0
$$

with $\bar{M}_{j}=$ Coker $u_{j}$ induces the embedding $\widetilde{M}_{j} \cong \bar{M}_{j}^{*} \stackrel{v_{j}^{*}}{\longrightarrow}\left(M_{j}^{\bullet}\right)^{*}=\widetilde{M}_{j}^{\bullet}$ required in Lemma 2.7 (i). Moreover, if $i \preceq j$ in $I$, then according to Lemma 2.8 (ii) there is a commutative diagram

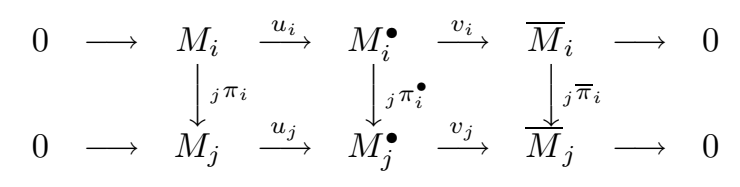

By the above equalities the dual diagram defines just the commutative diagram $(2.8)$ for the system $D^{\bullet}(\mathbf{M})=\left(\widetilde{M}_{i}\right)_{i \in I} \bullet$. This proves that $D^{\bullet}(\mathbf{M})=\left(\widetilde{M}_{i}\right)_{i \in I} \bullet$ is an object of $(I, \mathfrak{Z})^{\bullet}$-spr, as we required. 
Since there is a natural isomorphism $\left((I, \mathfrak{Z})^{\bullet}\right)^{\bullet} \cong(I, \mathfrak{Z})$ of posets with zerorelations, then the above construction applied to $(I, \mathfrak{Z})^{\bullet}$ defines the inverse reflection duality functor $D^{\bullet}:(I, \mathfrak{Z})^{\bullet}$-spr $\longrightarrow(I, \mathfrak{Z})$-spr.

Let us summarize the main facts about the reflection dualities in the following proposition.

Proposition 2.20. Let $(I, \mathfrak{Z})$ be a poset with zero-relations and let $(I, \mathfrak{Z})^{\bullet}$ be its reflection-dual poset with zero-relations (2.17). Then the following statements hold.

(a) There is an isomorphism $\left((I, \mathfrak{Z})^{\bullet}\right)^{\bullet} \cong(I, \mathfrak{Z})$ of posets with zero-relations.

(b) If $v \in \mathbb{N}^{I}$ is given and $v^{\bullet} \in \mathbb{N}^{I^{\bullet}}$ is such that $v_{j}^{\bullet}=v_{j}$ for $j \in I \backslash \max I$, and $v_{j}^{\bullet}=v_{p}$ for $j=p^{-}, p \in \max I$, then $q_{(I, \mathfrak{Z})}\left(v^{\bullet}\right)=q_{(I, \mathfrak{Z})}(v)$.

(c) The reflection duality functors (2.19) are dualities of categories inverse to each other. Moreover, they have the following properties:

(i) A sequence $0 \rightarrow \mathbf{M} \rightarrow \mathbf{N} \rightarrow \mathbf{L} \rightarrow$ is exact in the category $(I, \mathfrak{Z})$-spr if and only if $0 \rightarrow D^{\bullet}(\mathbf{M}) \rightarrow D^{\bullet}(\mathbf{N}) \rightarrow D^{\bullet}(\mathbf{L}) \rightarrow$ is an exact sequence in the category $(I, \mathfrak{Z})^{\bullet}$-spr.

(ii) The functor $D^{\bullet}$ carries relative injective objects to projective objects.

(iii) If $\mathbf{M}=\left(M_{j}\right)_{j \in I}$ is an object of $(I, \mathfrak{Z})$-spr and $\operatorname{dim} \mathbf{M}=\left(\operatorname{dim}_{K} M_{j}\right)_{j \in I}$, then

$$
\operatorname{dim} D^{\bullet}(\mathbf{M})=\mathbf{s}^{\bullet}(\operatorname{dim} \mathbf{M})
$$

where $\mathbf{s}^{\bullet}: \mathbb{Z}^{I} \longrightarrow \mathbb{Z}^{\bullet} \cong \mathbb{Z}^{I}$ is the group isomorphism defined by the formula

$$
\mathbf{s}^{\bullet}(w)_{j}= \begin{cases}-w_{j}+\sum_{j \prec p \in \max I} w_{p} & \text { if } j \in I \backslash \max I, \\ w_{p} & \text { if } j=p^{-}, p \in \max I .\end{cases}
$$

(d) The category $(I, \mathfrak{Z})$-spr is of tame (resp. wild) representation type if and only if the category $(I, \mathfrak{Z})^{\bullet}$-spr is of tame representation type.

Proof. Statements (a) and (b) follow from the definitions. For (b) we note that the relation $j \prec p \in \max I$ holds in $I$ and $(j, p) \notin \mathfrak{Z}$ if and only if the relation $j \prec p^{-} \in \max I^{\bullet}$ holds in $I^{\bullet}$ and $\left(j, p^{-}\right) \notin \mathfrak{Z}^{\bullet}$.

(c) For any $j \in I$ look at the exact sequence $0 \rightarrow M_{j} \stackrel{u_{j}}{\longrightarrow} M_{j}^{\bullet} \stackrel{v_{j}}{\longrightarrow} \bar{M}_{j} \rightarrow 0$ with $\bar{M}_{j}=$ Coker $u_{j}$. Since the $K$-dual to the map $u_{j}$ induces the embedding

$$
\widetilde{M}_{j} \cong \bar{M}_{j}^{*} \stackrel{v_{j}^{*}}{\longrightarrow}\left(M_{j}^{\bullet}\right)^{*}=\widetilde{M}_{j}^{\bullet}
$$

required in Lemma 2.8 (i), then by applying the definition of $D^{\bullet}$ to $D^{\bullet}(\mathbf{M})=$ $\left(\widetilde{M}_{j}\right)_{j \in I}$ we easily conclude that $D^{\bullet}\left(D^{\bullet}(\mathbf{M})\right) \cong \mathbf{M}$, and the isomorphism is functorial with respect to morphisms $\mathbf{M} \rightarrow \mathbf{N}$.

The proof of (i) and (ii) is routine, we leave it to the reader. For the proof of (iii) we recall from $(*)$ and $(* * *)$ above that $\widetilde{M}_{p^{-}}=M_{p}^{*}$ for $p \in \max I$ and $\widetilde{M}_{j} \cong \bar{M}_{j}^{*}$, $\widetilde{M}_{j}^{\bullet}=\left(M_{j}^{\bullet}\right)^{*}=\bigoplus_{\substack{\mathfrak{c} \mathfrak{j}_{j, p) \notin \mathfrak{Z}} \\ \text { max } I}} M_{p}^{*}$ for $j \in I \backslash \max I$, where $D^{\bullet}(\mathbf{M})=\left(\widetilde{M}_{j}\right)_{j \in I}$. Let $w=\operatorname{dim} \mathbf{M}=\left(\operatorname{dim}_{K} M_{j}\right)_{j \in I}$. It follows that $\operatorname{dim}_{K} \widetilde{M}_{p^{-}}=\operatorname{dim}_{K} M_{p}=\mathbf{s}^{\bullet}(w)_{p^{-}}$ for $p \in \max I$. In view of the exact sequence (*) above, given $j \in I \backslash \max I$ we get

$$
\begin{aligned}
\operatorname{dim}_{K} \widetilde{M}_{j} & =\operatorname{dim}_{K} \bar{M}_{j}=-\operatorname{dim}_{K} M_{j}+\operatorname{dim}_{K} M_{j}^{\bullet} \\
& =-\operatorname{dim}_{K} M_{j}+\sum_{\substack{j \preceq p \in \max I \\
(j, p) \notin \mathfrak{Z}}} \operatorname{dim}_{K} M_{p}=-w_{j}+\sum_{\substack{j \preceq p \in \max I \\
(j, p) \notin \mathfrak{Z}}} w_{p}=\mathbf{s}^{\bullet}(w)_{j} .
\end{aligned}
$$


This proves (iii) and finishes the proof of (c).

(d) Assume to the contrary that the category $(I, \mathfrak{Z})$-spr is of tame representation type, whereas the category $(I, \mathfrak{Z})^{\bullet}$-spr is not of tame representation type. Since the tame-wild dichotomy holds, then $(I, \mathfrak{Z})^{\bullet}$-spr $\cong \bmod _{\mathrm{sp}} K(I, \mathfrak{Z})$ is of wild representation type and there exists a representation embedding functor $F: \bmod \Gamma_{3}(K) \rightarrow$ $(I, \mathfrak{Z})^{\bullet}$-spr in the sense of [34], where

$$
\Gamma_{3}(K)=\left(\begin{array}{cc}
K & K^{3} \\
0 & K
\end{array}\right)
$$

is a generalized Kronecker $K$-algebra. Since $\Gamma_{3}(K)$ is self-dual and according to (c) the functor $D^{\bullet}$ is an exact equivalence of categories, then the composed functor

$$
\begin{aligned}
\bmod \Gamma_{3}(K) \stackrel{D}{\longrightarrow}\left(\bmod \Gamma_{3}(K)^{\mathrm{op}}\right)^{\mathrm{op} \cong} & \left(\bmod \Gamma_{3}(K)\right)^{\mathrm{op}} \\
& F^{\mathrm{op}} \downarrow \\
& \left((I, \mathfrak{Z})^{\bullet}-\mathrm{spr}\right)^{\mathrm{op}} \stackrel{D^{\bullet}}{\longrightarrow}(I, \mathfrak{Z}) \text {-spr }
\end{aligned}
$$

is a representation embedding functor, where $D=\operatorname{Hom}_{K}(-, K)$ is the standard duality functor. It follows from [34, Theorem 2.7] that the category $(I, \mathfrak{Z})$-spr is of wild representation type, and according to the tame-wild dichotomy the category $(I, \mathfrak{Z})$-spr is not of tame representation type, contrary to our assumption. The remaining part of (d) follows from the tame-wild dichotomy (see [9], 39]). This finishes the proof.

Remark 2.21. It follows from [30, Proposition 2.5(c) and (2.6)] or from a straightforward analysis that the reflection duality functor $D^{\bullet}(2.19)$ can be alternatively described as follows.

Any object $\mathbf{M}$ of $(I, \mathfrak{Z})$-spr can be viewed as an object of the category $(\widehat{I}, \widehat{\mathfrak{Z}})$-spr via the obvious embedding functor $(I, \mathfrak{Z})$-spr $\subseteq(\widehat{I}, \widehat{\mathfrak{Z}})$-spr $\cong \bmod _{\mathrm{sp}} K(\widehat{I}, \widehat{\mathfrak{Z}})$. It is easy to see that the injective envelope $\widehat{E}(\mathbf{M})$ of $\mathbf{M}$ in $\bmod K(\widehat{I}, \widehat{\mathfrak{Z}})$ is a socle projective module and is isomorphic to an object of $(\widehat{I}, \widehat{\mathfrak{Z}})$-spr. Consider the short exact sequence $0 \rightarrow \mathbf{M} \rightarrow \widehat{E}(\mathbf{M}) \rightarrow \overline{\mathbf{M}} \rightarrow 0$ in $\bmod K(I, \mathfrak{Z}) \stackrel{\simeq}{\longrightarrow} \operatorname{rep}_{K}(I, \mathfrak{Z})$ (see (2.3)). It is clear that $\bar{M}_{p}=0$ for all $p \in \max \widehat{I}$, and therefore the system $\overline{\mathbf{M}}^{*}=\left(\bar{M}_{j}^{*}\right)$ of $K$-dual vector spaces $\bar{M}_{j}^{*}$ is a peak $(I, \mathfrak{Z})^{\bullet}$-space isomorphic with $D^{\bullet}(\mathbf{M})$.

Remark 2.22. The class of multi-peak posets with zero-relations defined above is the smallest subclass in the class of all multi-peak bound quivers [30] containing multi-peak posets without zero-relations and closed under the reflection duality operation (2.17).

\section{A Reduction to Two-Peak Poset Representations}

With any $D$-order $\Lambda^{\bullet}(1.3)$ we associate in (3.3) below (see [40, Section 4]) a two-peak poset $\left(I_{\Lambda^{\bullet}}^{*+}, \mathfrak{Z}\right)$ with zero-relations, and we shall reduce the study of the category latt $\left(\Lambda^{\bullet}\right)$ to the study of the category $\left(I_{\Lambda^{\bullet}}^{*+}, \mathfrak{Z}\right)$-spr.

Suppose that $\Lambda, \Lambda_{1}, \Lambda_{2}$ and $\Lambda_{3}$ are tiled $D$-orders in (1.2). In order to define $\left(I_{\Lambda}^{*+}, \mathfrak{Z}_{\Lambda} \bullet\right)$ we consider the poset $\left(I_{\Lambda} ; \preceq\right)$ (see [45]), where

$$
I_{\Lambda}=\{1, \ldots, n\} \quad \text { and } \quad i \prec j \Leftrightarrow_{i} D_{j}=D .
$$


First we associate with $\Lambda^{\bullet}$ the combinatorial object

$$
I_{\Lambda} \bullet, \sigma=\left(I_{\Lambda}, \preceq, I^{\prime}, C, I^{\prime \prime}, \sigma: I^{\prime} \rightarrow I^{\prime \prime}\right)
$$

where $\left(I_{\Lambda}, \preceq\right)$ is the poset $(3.1), C=I_{\Lambda_{3}}=\left\{n_{1}+1 \prec \ldots \prec n_{1}+n_{3}-1 \prec n_{1}+n_{3}\right\}$, $I^{\prime}=I_{\Lambda_{1}}=\left\{1,2, \ldots, n_{1}\right\}$ and $I^{\prime \prime}=I_{\Lambda_{2}}=\left\{n_{1}+n_{3}+1, \ldots, n-1, n\right\}$ are viewed as subposets of $I_{\Lambda}$ such that $I_{\Lambda}=I^{\prime} \cup C \cup I^{\prime \prime}$ is a splitting decomposition of $I_{\Lambda}$ in the sense of [32. Section 8.1], and $\sigma: I^{\prime} \rightarrow I^{\prime \prime}$ is the poset isomorphism defined by the formula $\sigma(j)=n_{1}+n_{3}+j$. It is clear that $I_{\Lambda} \cdot \sigma$ is a bipartite stratified poset in the sense of 29], 31] and 32, Section 17.8], or a completed poset in the sense of 22 .

Let $C^{\prime}=\left\{c^{\prime} ; c \in C\right\}$ be a chain isomorphic with $C$. We construct two one-peak enlargements

$$
\left(C \cup I^{\prime \prime}\right)^{*}=C \cup I^{\prime \prime} \cup\{*\} \quad \text { and } \quad\left(I^{\prime} \cup C\right)^{+}=I^{\prime} \cup C^{\prime} \cup\{+\}
$$

of the posets $C \cup I^{\prime \prime}$ and $I^{\prime} \cup C \equiv I^{\prime} \cup C^{\prime}$ by the unique maximal points $*$ and + , and by the new relations $i \prec *$ and $s \prec+$ for all $i \in C \cup I^{\prime \prime}$ and all $s \in I^{\prime} \cup C^{\prime}$.

We associate with the $D$-order $\Lambda^{\bullet}(1.3)$ the two-peak poset with zero-relations

$$
\left(I_{\Lambda}^{*+}, \mathfrak{Z}_{\Lambda} \bullet\right)=\left(\left(C \cup I^{\prime \prime}\right)^{*} \cup_{I^{\prime \prime}} \equiv I^{\prime}\left(I^{\prime} \cup C\right)^{+}, \mathfrak{Z}_{\Lambda} \bullet\right)
$$

where $I_{\Lambda}^{*+}$ is obtained from the disjoint union $\left(C \cup I^{\prime \prime}\right)^{*} \cup\left(I^{\prime} \cup C\right)^{+}$of $\left(C \cup I^{\prime \prime}\right)^{*}$ and $\left(I^{\prime} \cup C\right)^{+}$by making the identification $j \equiv \sigma(j)$ for any element $j \in I^{\prime} \subseteq\left(I^{\prime} \cup C\right)^{+}$. The set $\mathfrak{Z}_{\Lambda} \bullet$ consists of all a pairs $\left(c, c_{1}^{\prime}\right)$ such that $c \in C \subseteq\left(C \cup I^{\prime \prime}\right)^{*}, c_{1}^{\prime} \in C^{\prime} \subseteq$ $\left(I^{\prime} \cup C\right)^{+}$and the relations $c \prec s, \sigma(s) \prec c_{1}$ hold in $I_{\Lambda}$ for some $s \in I^{\prime}$. Here we use the convention $+^{\prime}=+$.

It is easy to see that $I_{\Lambda^{\bullet}}^{*+}$ is a poset and $\max I_{\Lambda^{\bullet}}^{*+}=\{*,+\}$. We call $\left(I_{\Lambda^{\bullet}}^{*+}, \mathfrak{Z}_{\Lambda^{\bullet}}\right)$ a poset with zero-relations associated with the $D$-order $\Lambda^{\bullet}$.

Now we are able to prove our main reduction theorem.

Theorem 3.4. Let $K$ be an algebraically closed field, $D$ a complete discrete valuation domain which is a $K$-algebra, and $\mathfrak{p}$ is the unique maximal ideal of $D$. We assume that $D / \mathfrak{p} \cong K$. Let $\Lambda$ be the D-order (1.1) with the three-partition (1.2) and $\Lambda_{1}=\Lambda_{2} \subseteq \mathbb{M}_{n_{1}}(D), \Lambda_{3} \subseteq \mathbb{M}_{n_{3}}(D)$ and $n_{1}, n_{3}$ as in Section 1. Let $\Lambda^{\bullet}$ be the subamalgam D-order (1.3) and let $\left(I_{\Lambda^{\bullet}}^{*+}, \mathfrak{Z}_{\Lambda^{\bullet}}\right)$ be the two-peak poset with zero-relations (3.3) associated with $\Lambda^{\bullet}$.

(a) The Tits quadratic forms $q_{\Lambda} \bullet$ (1.4) and $q_{\left(I_{\Lambda}^{*+}, \mathfrak{z}_{\Lambda} \bullet\right)}$ in (2.13) coincide.

(b) There exists an additive reduction functor

$$
\mathbb{H}: \operatorname{latt}\left(\Lambda^{\bullet}\right) \longrightarrow\left(I_{\Lambda}^{*+}, \mathfrak{Z}_{\Lambda} \bullet\right)-\operatorname{spr} \cong \bmod _{\mathrm{sp}} K\left(I_{\Lambda}^{*+}, \mathfrak{Z}_{\Lambda} \bullet\right)
$$

with the following properties:

(i) $\mathbb{H}$ is full, reflects isomorphisms and preserves the indecomposability.

(ii) The image $\operatorname{Im} \mathbb{H}$ of $\mathbb{H}$ consists up to isomorphism of all objects of the category $\left(I_{\Lambda^{\bullet}}^{*+}, \mathfrak{Z}\right)$-spr having no direct summand of one of the following two types:

- the simple projective representation $P_{*}=e_{*} K\left(I_{\Lambda^{*}}^{*}, \mathfrak{Z}\right)$ corresponding to the peak idempotent $e_{*}$, and

- any of the hereditary sp-injective representations $\mathbf{H}_{n_{3}}^{-} \hookrightarrow \mathbf{H}_{n_{3}-1}^{-} \hookrightarrow \cdots \hookrightarrow \mathbf{H}_{0}^{-}$ defined in [40, (4.12)].

(iii) $\mathbb{H}$ preserves and reflects tame representation type, wild representation type, and the polynomial growth property; that is, $\operatorname{latt}\left(\Lambda^{\bullet}\right)$ is of tame representation 
type (resp. wild, or of polynomial growth) if and only if $\left(I_{\Lambda}^{*+}, \mathfrak{Z}_{\Lambda} \bullet\right)$-spr is of tame representation type (resp. wild, or of polynomial growth).

Proof. Statement (a) follows by a straightforward analysis.

(b) We take for the functor $\mathbb{H}$ the reduction functor constructed in 40 Definition 4.11] and defined to be the composed functor

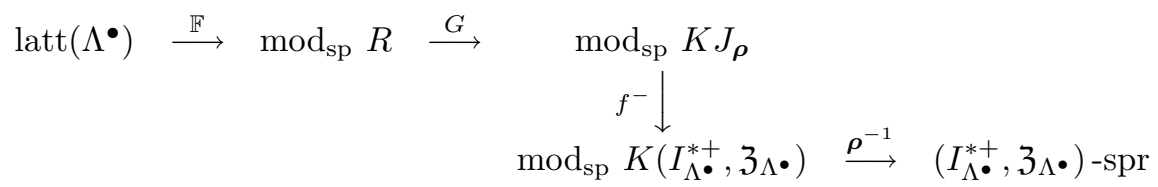

where $\boldsymbol{\rho}, f^{-}, G$ and $\mathbb{F}$ are the functors shown in (2.9), (3.7), (3.9) and in the diagram (3.11) below, and are defined as follows.

$1^{\circ}$ The functor $\mathbb{F}$. Consider the finite dimensional $K$-algebra

$$
R=\left(\begin{array}{cc}
\Lambda \bullet / \pi & \Gamma / \boldsymbol{\pi} \\
0 & \Gamma / \boldsymbol{\pi}
\end{array}\right),
$$

where $\Gamma=\mathbb{M}_{n}(D)$ and $\boldsymbol{\pi}=\mathbb{M}_{n}(\mathfrak{p})$. Note that $\Gamma$ is a hereditary $D$-order containing $\Lambda^{\bullet}$ and $\boldsymbol{\pi}$ is a two-sided ideal contained in the Jacobson radical $\operatorname{rad}(\Gamma)$ of $\Gamma$. It is also an ideal of $\Lambda^{\bullet}$ contained in $\operatorname{rad}\left(\Lambda^{\bullet}\right)$. It is easy to see that $R$ is a right peak $K$-algebra, that is, $R$ has a unique simple right ideal $P_{*}$ up to isomorphism (see [32]). We take for $\mathbb{F}$ the reduction functor

$$
\mathbb{F}: \operatorname{latt}\left(\Lambda^{\bullet}\right) \longrightarrow \bmod _{\mathrm{sp}} R
$$

defined [11] and 27] by the formula $\mathbb{F}(X)=(X / X \boldsymbol{\pi}, X \Gamma / X \boldsymbol{\pi}, u)$, where $X \Gamma$ is the $\Gamma$-submodule of $X \otimes_{D} F$ generated by $X$ (see [24]), $F=D_{0}$ is the field of fractions of $D$ and $u: X / X \boldsymbol{\pi} \rightarrow X \Gamma / X \boldsymbol{\pi}$ is the $\Lambda / \boldsymbol{\pi}$-monomorphism induced by the natural monomorphism $X \hookrightarrow X \Gamma$. We view $\mathbb{F}(X)$ as a right $R$-module in a natural way (see [11] and [27]).

By [11] and [27], the reduction functor $\mathbb{F}$ is full, reflects isomorphisms, preserves indecomposability, and Im $\mathbb{F}$ contains up to isomorphism all indecomposable objects of $\bmod _{\mathrm{sp}} R$ except from the unique simple right ideal $P_{*}$ of $R$.

It follows from [39, Theorem 7.19] that $\mathbb{F}$ preserves and reflects tame representation type, wild representation type, and the polynomial growth property. For note that [39, Theorem 7.19] applies, because in the case we consider here $\Gamma / \boldsymbol{\pi}$ is a simple $K$-algebra and according to [39, Proposition 4.5] the category $\bmod _{\mathrm{sp}} R$ is equivalent with the category $\bmod { }_{p r} R$ of projectively adjusted $R$-modules.

$2^{\circ}$ The functor $G$. Let $J=I_{\Lambda}^{*}=I_{\Lambda} \cup\{*\}$ be the poset obtained from $I_{\Lambda}$ by adding the unique maximal element $*$ with new relations $i \prec *$ for all $i \in I_{\Lambda}$. Consider the set

$$
\mathbf{\Delta} J:=\{(i, j) ; i \preceq j \text { in } J\} \subseteq J \times J
$$

and define a binary equivalence relation $\boldsymbol{\rho}$ on $\boldsymbol{\Lambda} J$ by setting

$$
(i, j) \boldsymbol{\rho}(s, t) \Leftrightarrow(i, j)=(s, t) \text { or } i, s \in I^{\prime}=I_{\Lambda_{1}}, j, t \in I^{\prime \prime}=I_{\Lambda_{2}}, j=\sigma(i), t=\sigma(s),
$$

where $\sigma: I^{\prime} \rightarrow I^{\prime \prime}$ given by $\sigma(i)=i+n_{1}+n_{3}$ is a poset isomorphism. Then we have defined a bipartite stratified poset

$$
J_{\rho}=(J, \rho)
$$


in the sense of [29] and [31, Definition 4.1]. The bipartition $J=J^{\prime}+C+J^{\prime \prime \prime}$ is given by taking $J^{\prime}=I^{\prime}, C=I_{\Lambda_{3}}$ and $J^{\prime \prime \prime}=\left(I^{\prime \prime}\right)^{*}$. We recall from [29] and [31] that the incidence $K$-algebra of $J_{\boldsymbol{\rho}}$ is the subalgebra $K J_{\boldsymbol{\rho}}$ of $K J$ consisting of all matrices $\lambda=\left[\lambda_{p q}\right]_{p, q \in J}$ such that $\lambda_{i j}=\lambda_{s t}$ if $(i, j),(s, t) \in \mathbf{\Lambda} J$ and $(i, j) \boldsymbol{\rho}(s, t)$. It was shown in 31. that $K J_{\rho}$ is a basic right peak $K$-algebra and the right socle of $K J_{\rho}$ is isomorphic to a direct sum of the simple projective right ideal $P_{*}^{\prime}=e_{*} K J_{\rho}$, called a right peak of $K J_{\rho}$. A simple analysis shows that the algebra $R$ defined above is Morita equivalent with the incidence algebra $K J_{\rho}$. The idea of the proof of this fact is explained by Example 3.9 in [38, p. 95]. We define a $K$-linear functor

$$
G: \bmod _{\mathrm{sp}} R \simeq \bmod _{\mathrm{sp}} K J_{\rho}
$$

to be the Morita equivalence restricted to socle projective modules. It is clear that $G$ preserves and reflects finite representation type, tameness, wildness, and the polynomial growth property.

$3^{\circ}$ The functor $f^{-}$. Let $(Q, \Omega)=\left(Q\left(J_{\boldsymbol{\rho}}\right), \Omega\left(J_{\boldsymbol{\rho}}\right)\right)$ be the bound quiver associated

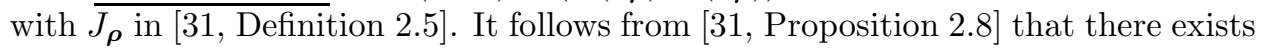
a $K$-algebra isomorphism $K(Q, \Omega) \cong K J_{\rho}$. Let

$$
f:(\widetilde{Q}, \widetilde{\Omega}) \longrightarrow(Q, \Omega)
$$

be the bound quiver Galois covering [31, (3.1)] of $(Q, \Omega)$. It follows from [31, Proposition 3.8] that $f$ is a universal covering with the covering group $\mathbb{Z}$. Moreover, it follows from the construction that

$$
\left(I_{\Lambda}^{*+}, \mathfrak{Z}_{\Lambda} \bullet\right) \cong J_{\boldsymbol{\rho}}^{*+},
$$

where $J_{\boldsymbol{\rho}}^{*+}$ is the two-peak bound subquiver of the quiver $(\widetilde{Q}, \widetilde{\Omega})$ associated with $J_{\rho}$ in 31 (4.3)] and $\left(I_{\Lambda}^{*+}, \mathfrak{Z}_{\Lambda} \bullet\right)$ is the poset with zero-relations associated with $\Lambda^{\bullet}$ by the formula (3.3). By [31 Theorem 4.19] the push-down functor $f_{\lambda}$ : $\bmod K(\widetilde{Q}, \widetilde{\Omega}) \rightarrow \bmod K(Q, \Omega)$ induces the push-down functor

$$
\bmod _{\mathrm{sp}} K(\widetilde{Q}, \widetilde{\Omega}) \stackrel{f_{s p}}{\longrightarrow} \bmod _{\mathrm{sp}} K(Q, \Omega) \cong \bmod _{\mathrm{sp}} K J_{\boldsymbol{\rho}}
$$

and we get the following diagram:

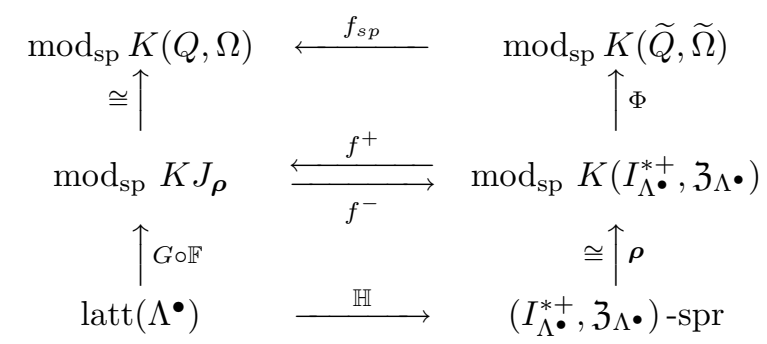

where (under the identification $\left.J_{\boldsymbol{\rho}}^{*+} \equiv\left(I_{\Lambda}^{*+}, \mathfrak{Z}_{\Lambda} \bullet\right)\right) f^{+}$is the glueing functor 31 (4.14)], $\Phi=T_{v} \circ L_{\xi}$ is the embedding defined in [31, Proposition 4.23], $f^{-}$is the section functor [31, (5.1)], and $\boldsymbol{\rho}$ is the equivalence of categories defined in (2.9). The idea of this construction is explained by Example 3.9 in [38, p. 95].

According to [31 Proposition 4.23], the category $\bmod _{\mathrm{sp}} K(\widetilde{Q}, \widetilde{\Omega})$ is locally coordinate support finite and every indecomposable module of $\bmod _{\mathrm{sp}} K(\widetilde{Q}, \widetilde{\Omega})$ is contained in the image of the functor $\Phi$, up to a $\mathbb{Z}$-shift. It then follows from [31] Theorem 4.27] and the main results of Dowbor and Skowroński in [7] and [8] (see 
also [6]) that the push-down functor $f_{s p}$, and hence the functors $f^{+}$and $f^{-}$as well, preserve and reflect tameness, wildness and the polynomial growth property (apply 31, Proposition 5.4 and Theorem 5.8]).

Hence we easily conclude that the composed functor $\mathbb{H}(3.5)$ has the properties stated in (i) and (iii) of the theorem. Since the statement (ii) was proved in [40. Theorem 4.14], the proof of the theorem is complete.

Remark 3.12. (a) It follows from [40, Theorem 6.1] that the Auslander-Reiten quiver of the category $\left(I_{\Lambda^{\bullet}}^{*+}, \mathfrak{Z}_{\Lambda^{\bullet}}\right)$-spr has the form presented in Figure 3.13. If $\left(I_{\Lambda^{\bullet}}^{*+}, \mathfrak{Z}_{\Lambda^{\bullet}}\right)$-spr is of finite lattice type then the part $\mathcal{R}$ in Figure 3.13 is empty, $\mathcal{P}\left(\Lambda^{\bullet}\right)=\mathcal{I}\left(\Lambda^{\bullet}\right)$, and $\mathcal{P}\left(\Lambda^{\bullet}\right)$ is finite.

(b) In view of [40. Theorem 6.1] we have a description of the Auslander-Reiten quiver $\Gamma\left(\operatorname{latt}\left(\Lambda^{\bullet}\right)\right)$ of $\operatorname{latt}\left(\Lambda^{\bullet}\right)$. By applying the reduction functor

$$
\mathbb{H}: \operatorname{latt}\left(\Lambda^{\bullet}\right) \rightarrow\left(I_{\Lambda}^{*+}, \mathfrak{Z}_{\Lambda} \bullet\right)-\operatorname{spr}
$$

(3.6) the Auslander-Reiten quiver $\Gamma\left(\operatorname{latt}\left(\Lambda^{\bullet}\right)\right)$ is obtained from the AuslanderReiten quiver $\Gamma\left(\left(I_{\Lambda^{\bullet}}^{*+}, \mathfrak{Z}_{\Lambda^{\bullet}}\right)\right.$-spr $)$ of $\left(I_{\Lambda^{\bullet}}^{*+}, \mathfrak{Z}_{\Lambda^{\bullet}}\right)$-spr by the following two simple glueings:

$1^{\circ}$ The identification of a hereditary projective section

$$
\mathbf{P}_{n_{3}}^{+} \hookrightarrow \mathbf{P}_{n_{3}-1}^{+} \hookrightarrow \cdots \hookrightarrow \mathbf{P}_{0}^{+}
$$

of irreducible monomorphisms from the beginning of the unique preprojective component $\mathcal{P}\left(\Lambda^{\bullet}\right)$ in $\left(I_{\Lambda^{\bullet}}^{*+}, \mathfrak{Z}_{\Lambda^{\bullet}}\right)$-spr containing the simple projective module $\mathbf{P}_{n_{3}}^{+} \cong$ $e_{+} K\left(I_{\Lambda^{\bullet}}^{*+}, \mathfrak{Z}_{\Lambda^{\bullet}}\right)$ with a hereditary sp-injective section

$$
\mathbf{H}_{n_{3}}^{-} \hookrightarrow \mathbf{H}_{n_{3}-1}^{-} \hookrightarrow \cdots \hookrightarrow \mathbf{H}_{0}^{-}
$$

of irreducible monomorphisms from the end the unique preinjective component $\mathcal{I}\left(\Lambda^{\bullet}\right)$ in $\left(I_{\Lambda^{\bullet}}^{*+}, \mathfrak{Z}_{\Lambda^{\bullet}}\right)$-spr containing the injective envelope $\mathbf{H}_{0}^{-}$of the simple projective module $e_{*} K\left(I_{\Lambda}^{*+}, \mathfrak{Z}_{\Lambda} \bullet\right)$.

$2^{\circ}$ The identification of the simple projective module $P_{*}=e_{*} K\left(I_{\Lambda^{\bullet}}^{*+}, \mathfrak{Z}_{\Lambda^{\bullet}}\right)$ with the injective envelope $E\left(\mathbf{P}_{n_{3}}^{+}\right)$of the simple projective module

$$
\mathbf{P}_{n_{3}}^{+} \cong e_{+} K\left(I_{\Lambda}^{*+}, \mathfrak{Z}_{\Lambda} \bullet\right)
$$

in the category $\left(I_{\Lambda^{\bullet}}^{*+}, \mathfrak{Z}_{\Lambda} \bullet\right)-\operatorname{spr} \cong \bmod _{\mathrm{sp}} K\left(I_{\Lambda}^{*+}, \mathfrak{Z}_{\Lambda} \bullet\right)$.

The reader is referred to [40, Section 6] for details. The glueing procedure of quiver 3.13 is explained in Example 6.6 of [40] (see also [25, pp. 451-455] and [26]).

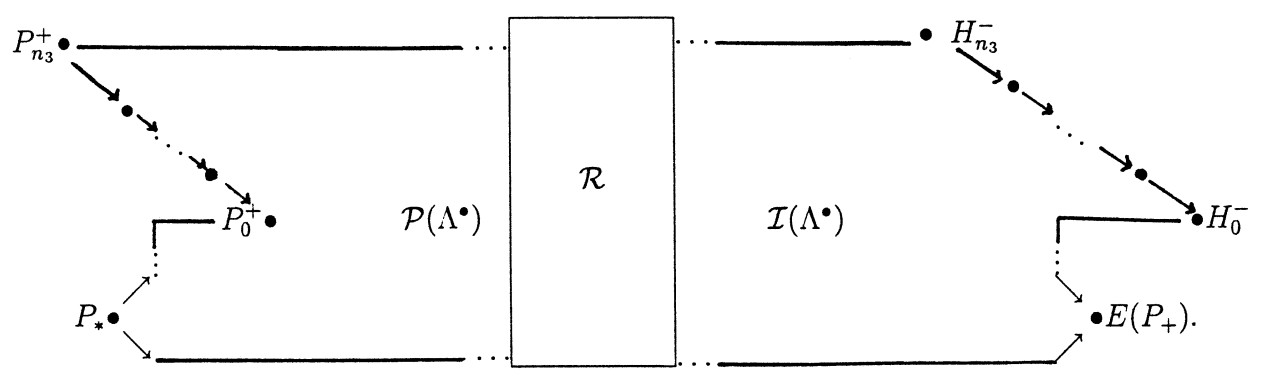

Figure 3.13. The shape of Auslander-Reiten quiver of the category $\left(I_{\Lambda}^{*+}, \mathfrak{Z}_{\Lambda} \bullet\right)-\operatorname{spr}$ 
We finish this section by the following useful result concerning the existence of preprojective components.

Proposition 3.14. Let $\left(I_{\Lambda}^{*+}, \mathfrak{Z}_{\Lambda} \bullet\right)$ be the poset with zero-relations (3.3) associated with the three-partite subamalgam $D$-order $\Lambda^{\bullet}(1.3)$.

(a) Every point of the poset with zero-relations $\left(I_{\Lambda}^{*+}, \mathfrak{Z}_{\Lambda} \bullet\right)$ is separating in the sense of Bongartz 4] (see also [13, 31, Section 4]).

(b) There exist a preprojective component $\widetilde{\mathcal{P}}_{\left(I_{\Lambda}^{*} \bullet, \mathfrak{Z}_{\Lambda} \bullet\right)}$ in prin $K\left(I_{\Lambda^{*}}^{*+}, \mathfrak{Z}_{\Lambda} \bullet\right)$ and a preprojective component $\mathcal{P}_{\left(I_{\Lambda}^{*+} \bullet \mathfrak{Z}_{\Lambda} \bullet\right)}$ in the category $\left(I_{\Lambda}^{*+}, \mathfrak{Z}_{\Lambda} \bullet\right)-\operatorname{spr}$ such that the adjustment functor (2.10)

$$
\boldsymbol{\theta}: \operatorname{prin} K\left(I_{\Lambda}^{*+}, \mathfrak{Z}_{\Lambda} \bullet\right) \longrightarrow\left(I_{\Lambda}^{*+}, \mathfrak{Z}_{\Lambda} \bullet\right)-\operatorname{spr}
$$

carries $\widetilde{\mathcal{P}}_{\left(I_{\Lambda}^{*+}, \mathfrak{z}_{\Lambda} \bullet\right)}$ to $\mathcal{P}_{\left(I_{\Lambda}^{*+}, \mathfrak{Z}_{\Lambda} \bullet\right)}$.

(c) The preprojective components $\widetilde{\mathcal{P}}_{\left(I_{\Lambda}^{* \bullet} \bullet \mathbf{3}_{\Lambda} \bullet\right)}$ and $\mathcal{P}_{\left(I_{\Lambda}^{*+}, \boldsymbol{Z}_{\Lambda} \bullet\right)}$ can be constructed by Algorithm 4.4 in [19].

Proof. The existence of a preprojective component $\widetilde{\mathcal{P}}_{\left(I_{\Lambda}^{*+}, \mathfrak{3}_{\Lambda} \bullet\right)}$ in the category prin $K\left(I_{\Lambda}^{*+}, \mathfrak{Z}_{\Lambda} \bullet\right)$ and statement (a) follow from [31, Proposition 4.9] applied to the bipartite stratified poset $I_{\Lambda} \bullet, \sigma(3.2)$, because the algebra $K\left(I_{\Lambda}^{*+}, \mathfrak{Z}_{\Lambda} \bullet\right)$ is obtained from $I_{\Lambda} \bullet, \sigma$ by a construction required in [31, Proposition 4.9] and the arguments of Bongartz [4] apply (see also [13] and [19, Algorithm 4.4]). By [23, Lemma 3.12, Theorem 3.13] and properties of the adjustment functor $\boldsymbol{\theta}$ proved there, the image $\mathcal{P}_{\left(I_{\Lambda}^{*} \bullet, \mathbf{Z}_{\Lambda} \bullet\right)}$ of $\widetilde{\mathcal{P}}_{\left(I_{\Lambda}^{*+}, \mathfrak{Z}_{\Lambda} \bullet\right)}$ under $\boldsymbol{\theta}$ is a preprojective component in the category $\left(I_{\Lambda}^{*+}, \mathfrak{Z}_{\Lambda} \bullet\right)$-spr. Note that the arguments given for [19, Algorithm 4.4] and in [32, Theorem 11.68 and Corollary 11.76] for the case of one-peak posets extend to our situation.

\section{Proof of Main Results}

Throughout this section $K$ is an algebraically closed field and $D$ is a complete discrete valuation domain which is a $K$-algebra such that $D / \mathfrak{p} \cong K$, where $\mathfrak{p}$ is the unique maximal ideal of $D$.

We start with the following useful reflection duality result.

Proposition 4.1. Let $\Lambda^{\bullet}$ be a subamalgam D-suborder (1.3) of the tiled order $\Lambda$ $(1.2)$, let $\Gamma^{\bullet}=\operatorname{rt}\left(\Lambda^{\bullet}\right)$ be the reflection transpose order $(1.7)$ of $\Lambda^{\bullet}$, let $\left(I_{\Lambda}^{*+}, \mathfrak{Z}_{\Lambda} \bullet\right)$ be the two-peak poset with zero-relations $(3.3)$, and let $\left(I_{\Lambda}^{*+}, \mathfrak{Z}_{\Lambda} \bullet\right)^{\bullet}$ be its reflection-dual (2.17). Then the following statements hold.

(a) There is a D-algebra isomorphism $\Gamma^{\bullet} \cong\left(\Lambda^{\bullet}\right)^{\mathrm{op}}$ and an isomorphism

$$
\left(I_{\Lambda}^{*+}, \mathfrak{Z}_{\Lambda} \bullet\right)^{\bullet} \cong\left(I_{\Gamma}^{*+}, \mathfrak{Z}_{\Gamma} \bullet\right)
$$

of two-peak posets with zero-relations.

(b) There exists a commutative diagram

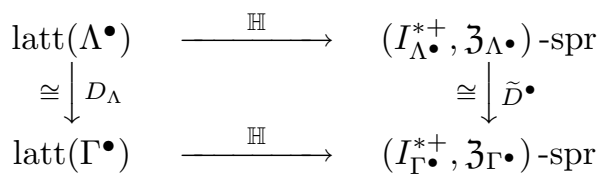


where $\mathbb{H}$ is the composed reduction functor $(3.6), D_{\Lambda}=\operatorname{Hom}_{D}(-, D)$ is the standard $D$-duality, and $\widetilde{D}^{\bullet}$ is the composed duality functor

$$
\left(I_{\Lambda}^{*+}, \mathfrak{Z}_{\Lambda} \bullet\right)-\operatorname{spr} \stackrel{D^{\bullet}}{\longrightarrow}\left(I_{\Lambda}^{*+}, \mathfrak{Z}_{\Lambda} \bullet\right)^{\bullet}-\operatorname{spr} \cong\left(I_{\Gamma}^{*+}, \mathfrak{Z}_{\Gamma} \bullet\right)-\operatorname{spr}
$$

induced by the reflection duality (2.19).

(c) The D-order $\Lambda^{\bullet}$ is of tame lattice type if and only if the $D$-order $\Lambda^{\bullet}$ is of tame lattice type.

Proof. Statements (a) and (b) follow directly from the definitions. The details are left to the reader. Statement (c) follows by applying the tame-wild dichotomy for $D$-orders proved in [9], because the arguments used in the proof of Proposition 2.20 (d) easily extend to our case.

We shall need the following two simple lemmas.

Lemma 4.3. Let $\Omega$ be a D-order in a semisimple $K$-algebra $C$ and let $e \in \Omega$ be an idempotent. Then e $\Omega e$ is a D-order in the semisimple $K$-algebra eCe, and the following statements hold.

(a) The functors

$$
\operatorname{latt}(e \Omega e) \underset{\operatorname{res}_{e}}{\stackrel{L_{e}}{\leftrightarrows}} \operatorname{latt}(\Omega)
$$

defined by the formulas $\operatorname{res}_{e}(X)=X e, L_{e}(Y)=\operatorname{Hom}_{e \Omega e}(\Omega e, Y)$ have the following properties:

(i) The functor $L_{e}$ is a fully faithful embedding, $\operatorname{res}_{e} L_{e} \cong \mathrm{id}$, and $L_{e}$ is right adjoint to $\mathrm{res}_{e}$, that is, there is a natural isomorphism

$$
\operatorname{Hom}_{\Omega}\left(X, L_{e}(Y)\right) \cong \operatorname{Hom}_{e \Omega e}\left(\operatorname{res}_{e}(X), Y\right)
$$

for every $\Omega$-lattice $X$ and every e $\Omega$-lattice $Y$.

(ii) The restriction functor $\mathrm{res}_{e}$ is exact, and $L_{e}$ is left exact and preserves the indecomposability.

(b) If the D-order $\Omega$ is of finite lattice type, then the D-order e $\Omega$ is of finite lattice type.

(c) If the D-order $\Omega$ is of tame lattice type (resp. tame of polynomial growth), then the D-order e $\Omega$ is of tame lattice type (resp. tame of polynomial growth).

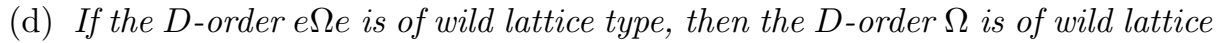
type.

Proof. Statement (a) is well-known and follows by the arguments applied in the proof of [32, Theorem 17.46]. The details are left to the reader. We only remark that the module $L_{e}(X)$ is finitely generated and $D$-torsionfree, if $X$ is finitely generated and $D$-torsionfree.

It follows from (a) that the functor $L_{e}$ carries indecomposable modules to indecomposable modules and carries nonisomomorphic modules to nonisomomorphic ones. Hence (b) easily follows.

(c) Assume that $\Omega$ is of tame lattice type and the functors

$$
(-) \otimes_{A} M^{(1)}, \ldots,(-) \otimes_{A} M^{(s)}: \operatorname{ind}_{1}(A) \longrightarrow \operatorname{latt}(\Omega)
$$

(1.8) form an almost parameterizing family for the category $\operatorname{ind}_{r}(\operatorname{latt}(\Omega))$ of indecomposable $\Omega$-lattices of $D$-rank $r$. Since the restriction functor $\operatorname{res}_{e}(-)=(-) e$ is 
exact, a simple analysis shows that the functors

$$
(-) \otimes_{A} M^{(1)} e, \ldots,(-) \otimes_{A} M^{(s)} e: \operatorname{ind}_{1}(A) \longrightarrow \operatorname{latt}(e \Omega e)
$$

form an almost parameterizing family for the category $\operatorname{ind}_{r}(\operatorname{latt}(e \Omega e))$ of indecomposable $e \Omega e$-lattices of $D$-rank $r$. This proves that $e \Omega e$ is of tame lattice type. The polynomial growth version follows in a similar way.

The statement (d) follows immediately from (c) by applying the tame-wild dichotomy for $D$-orders proved in $[9]$.

Lemma 4.4. Assume that $\Lambda \subseteq \Omega$ are D-orders in a semisimple $K$-algebra $C$.

(a) If $\Lambda$ is of finite lattice type, then $\Omega$ is of finite lattice type.

(b) If $\Lambda$ is of tame lattice type, then $\Omega$ is of tame lattice type.

(d) If $\Omega$ is of wild lattice type, then $\Lambda$ is of wild lattice type.

Proof. It is easy to check that the forgetful functor $\operatorname{res}_{\Lambda}: \operatorname{latt}(\Omega) \longrightarrow \operatorname{latt}(\Lambda)$ (associating with any $\Gamma$-module $X$ the vector space $X$ viewed as $\Lambda$-module) is full, faithful and exact (see [5, p. 532, Ex. 2]). Hence (a) and (c) easily follow. The statement (b) follows immediately from (c), because of the tame-wild dichotomy for $D$-orders proved in $[9]$.

Proof of Theorem 1.5. (a) $\Rightarrow(\mathrm{b})$. It follows from Theorem 3.4 (a) that the Tits quadratic forms $q_{\Lambda} \bullet(1.4)$ and $q_{\left(I_{\Lambda}^{*} \bullet, \mathfrak{Z}_{\Lambda} \bullet\right)}(z)=b_{\left(I_{\Lambda}^{*+}, \mathfrak{Z}_{\Lambda} \bullet\right)}(z, z)$ in $(2.13)$ coincide. Then the implication $(\mathrm{a}) \Rightarrow(\mathrm{b})$ follows from Theorem 3.4 (iii) and Theorem 2.14.

$(\mathrm{b}) \Rightarrow(\mathrm{d})$. Let $(L, \mathfrak{Z})$ be any of the two-peak posets with zero-relations listed in Theorem $1.5(\mathrm{~d})$. We claim that there exists a vector $v_{(L, \mathfrak{Z})} \in \mathbb{N}^{L}$ such that $q_{(L, \mathfrak{Z})}\left(v_{(L, \mathfrak{Z})}\right)<0$. In case $\mathfrak{Z}$ is empty the claim follows from [16, Theorem 1.3], because the two-peak posets without zero-relations listed in Theorem $1.5(\mathrm{~d})$ are the hypercritical ones presented in Table 1 of [16, pp. 509-511]. It remains to prove the claim if $(L, \mathfrak{Z})$ is the poset $\widehat{\mathcal{F}}_{4}$ with one zero-relation. Since obviously $\widehat{\mathcal{F}}_{4}=\widehat{\mathcal{F}}_{5}$ is reflection-dual to the poset $\widehat{\mathcal{F}}_{5}$, then we can take for $v_{\widehat{\mathcal{F}}_{4}}$ the vector $v_{\widehat{\mathcal{F}}_{5}}^{\bullet}$ defined in Proposition $2.20(\mathrm{~b})$, because it is shown there that $q_{\widehat{\mathcal{F}}_{4}}\left(v_{\widehat{\mathcal{F}}_{5}}^{\bullet}\right)=q_{\widehat{\mathcal{F}}_{5}}\left(v_{\widehat{\mathcal{F}}_{5}}\right)<0$. Since according to Theorem 3.4 (a) the quadratic forms $q_{\Lambda} \bullet$ and $q_{\left(I_{\Lambda}^{*+}, \mathfrak{Z}_{\Lambda} \bullet\right)}$ coincide, the implication $(\mathrm{b}) \Rightarrow(\mathrm{d})$ follows.

$(d) \Rightarrow(\mathrm{a})$. We consider three cases.

$\underline{\text { Case } 1^{\circ}} \cdot n_{3}=0$. It follows that the sets $C, C^{\prime}$ and $\mathfrak{Z}_{\Lambda} \bullet$ in the definition of $\left(I_{\Lambda^{\bullet}}^{*+}, \mathfrak{Z}_{\Lambda^{\bullet}}\right)(3.3)$ are empty. By condition $(\mathrm{d})$ of the theorem the two-peak poset $I_{\Lambda}^{*+}$ does not contain as a two-peak subposet the posets $\widehat{\mathcal{F}}_{0}^{2}$ and $\widehat{\mathcal{F}}_{0}^{3}$. Thus $I_{\Lambda}^{*+}$ is a peak subposet of a two-peak garland

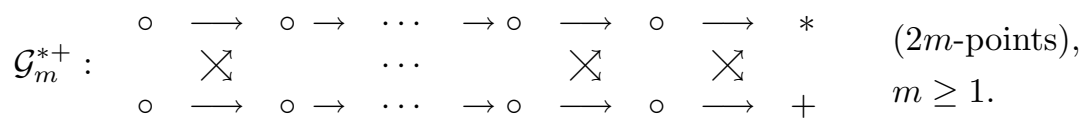

It follows from the proof of the implication $(\mathrm{c}) \Rightarrow(\mathrm{a})$ in [29, Proposition 4.13] or from [37, Theorem 5.2] (see also [36]) that the category $\mathcal{G}_{m}^{*+}$-spr is of tame representation type. Further, if $m \geq 3, \mathcal{G}_{m}^{*+}$-spr is of non-polynomial growth (see also [18] Lemma 3.1]). It follows from [40, Proposition 2.9] that the category $\left(I_{\Lambda}^{*+}, \mathfrak{Z}_{\Lambda} \bullet\right)$-spr is of tame representation type. Hence, in view of Theorem 3.4(iii), the category $\operatorname{latt}\left(\Lambda^{\bullet}\right)$ is of tame representation type, and (a) follows. 


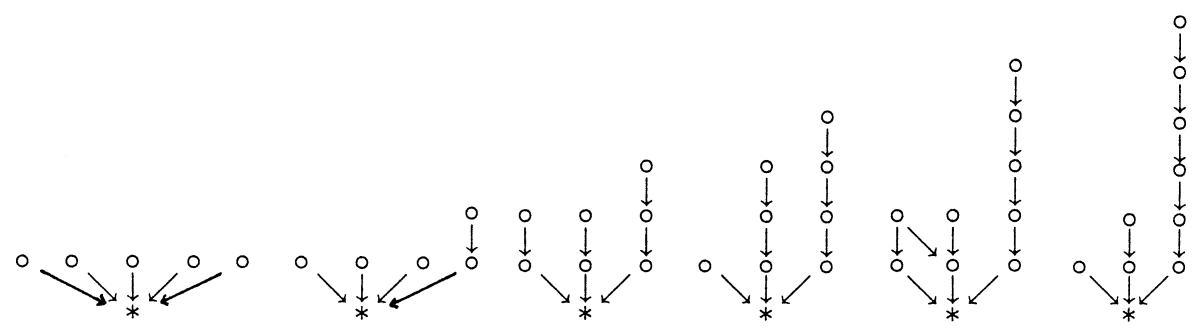

TABLE 4.6. One-peak enlargements of hypercritical posets of Nazarova

Case $2^{\circ} \cdot n_{3} \geq 1$ and the part $\mathcal{Y}$ of $\Lambda$ in (1.2) consists of matrices with coefficients in $\mathfrak{p}$. It follows from the definition of $\left(I_{\Lambda}^{*+}, \mathfrak{Z}_{\Lambda^{\bullet}}\right)(3.3)$ that the chains $C$ and $C^{\prime}$ are not empty, $C$ is incomparable with all elements of $I^{\prime} \equiv I^{\prime \prime}$, and the set $\mathfrak{Z}_{\Lambda} \bullet$ of zerorelations is empty. Hence we conclude that the poset $I^{\prime} \cong I^{\prime \prime}$ is linearly ordered, because otherwise the poset $C \cup I^{\prime \prime}$ contains a triple of incomparable points and therefore the poset $I_{\Lambda}^{*+}$ contains a two-peak subposet isomorphic with $\widehat{\mathcal{F}}_{0}^{1}$, contrary to the assumption (d).

This shows that in this case the two-peak poset $I_{\Lambda}^{*+}$ is thin in the sense of [18. Definition 3.1], and according to [18, Theorem 1.3] the following three statements are equivalent:

$\left(\mathrm{a}^{\prime}\right)$ The category $I_{\Lambda}^{*+}$-spr is of tame representation type.

$\left(\mathrm{b}^{\prime}\right)$ The Tits quadratic form $q_{I_{\Lambda}^{*+}}$ is weakly non-negative.

(c') The two-peak poset $I_{\Lambda}^{*+}$ associated with $\Lambda^{\bullet}$ in (3.3) does not contain as a twopeak subposet any of the hypercritical two-peak posets presented in [18. Table 1], and does not contain as a peak subposet any of the one-peak enlargements $\mathcal{N}_{1}^{*}$, $\mathcal{N}_{2}^{*}, \mathcal{N}_{3}^{*}, \mathcal{N}_{4}^{*}, \mathcal{N}_{5}^{*}, \mathcal{N}_{6}^{*}$ of hypercritical Nazarova posets [21] shown in Table 4.6 (see also [32, Theorem 15.3]).

Note that the poset $I_{\Lambda^{*}}^{*+} \backslash\left(I^{\prime} \cup\{*,+\}\right)$ is a disjoint union of two chains $C$ and $C^{\prime}$. Then a case by case inspection of the two peak posets in [17, Table 1] and [18, Table 1] shows that, for any three-partite subamalgam $D$-order $\Lambda^{\bullet}(1.3)$ such that the poset $I^{\prime}=I_{\Lambda_{1}}$ is linearly ordered, the two-peak poset $I_{\Lambda^{\bullet}}^{*+}$ does not contain as a peak subposet any of the one-peak enlargements $\mathcal{N}_{1}^{*}, \mathcal{N}_{2}^{*}, \mathcal{N}_{3}^{*}, \mathcal{N}_{4}^{*}, \mathcal{N}_{5}^{*}, \mathcal{N}_{6}^{*}$ of hypercritical Nazarova posets, and $I_{\Lambda}^{*+}$ could contain at most the nine hypercritical posets $\widehat{\mathcal{F}}_{1}^{1}, \widehat{\mathcal{F}}_{1}^{2}, \widehat{\mathcal{F}}_{2}, \widehat{\mathcal{F}}_{3}^{1}, \widehat{\mathcal{F}}_{3}^{2}, \widehat{\mathcal{F}}_{5}, \widehat{\mathcal{F}}_{6}, \widehat{\mathcal{F}}_{7}$ and $\widehat{\mathcal{F}}_{8}$ listed in Theorem 1.5 from the 41 posets presented in [18. Table 1]. It then follows that under the assumption we make in Case $2^{\circ}$, the condition (d) of Theorem 1.5 is equivalent with the condition $\left(\mathrm{c}^{\prime}\right)$ above and therefore $(\mathrm{d})$ implies the tameness of $I_{\Lambda^{\bullet}}^{*+}$-spr. Since $\mathfrak{Z}_{\Lambda} \bullet$ is empty, then in view of Theorem 3.4, this implies that the order $\Lambda^{\bullet}$ is of tame lattice type, and (a) follows.

$\underline{\text { Case } 3^{\circ}} \cdot n_{3} \geq 1$ and the part $\mathcal{X}$ of $\Lambda$ in (1.2) consists of matrices with coefficients in $\mathfrak{p}$. Let $\Gamma^{\bullet}=\operatorname{rt}\left(\Lambda^{\bullet}\right)$ be the reflection transpose of $\Lambda^{\bullet}$ (see (1.7)). Since the part $\mathcal{X}$ of $\Lambda$ in (1.2) consists of matrices with coefficients in $\mathfrak{p}$, then the corresponding part $\mathcal{Y}$ of $\Gamma$ in its three-partition (1.2) consists of matrices with coefficients in $\mathfrak{p}$ and by the arguments in Case $2^{\circ}$ applied to $\Gamma^{\bullet}$ the set $\mathfrak{Z}_{\Gamma}$ • is empty. It follows from Proposition 4.1 that $I_{\Gamma^{\bullet}}^{*+}=\left(I_{\Gamma^{\bullet}}^{*+}, \mathfrak{Z}_{\Gamma^{\bullet}}\right) \cong\left(I_{\Lambda^{\bullet}}^{*+}, \mathfrak{Z}_{\Lambda} \bullet\right)^{\bullet}$, and according to $(2.19)$ there 
exists a reflection duality functor

$$
D^{\bullet}: I_{\Gamma}^{*+}-\operatorname{spr} \longrightarrow\left(I_{\Lambda}^{*+}, \mathfrak{Z}_{\Lambda}^{\bullet}\right)^{\bullet} \text {-spr . }
$$

Since the two-peak poset $\left(I_{\Lambda}^{*+}, \mathfrak{Z}_{\Lambda} \bullet\right)$ with zero-relations does not contains any of the following thirteen hypercritical posets with zero-relations $\widehat{\mathcal{F}}_{0}^{1}, \widehat{\mathcal{F}}_{0}^{2}, \widehat{\mathcal{F}}_{0}^{3}, \widehat{\mathcal{F}}_{1}^{1}, \widehat{\mathcal{F}}_{1}^{2}$, $\widehat{\mathcal{F}}_{2}, \widehat{\mathcal{F}}_{3}^{1}, \widehat{\mathcal{F}}_{3}^{2}, \widehat{\mathcal{F}}_{4}, \widehat{\mathcal{F}}_{5}, \widehat{\mathcal{F}}_{6}, \widehat{\mathcal{F}}_{7}$ and $\widehat{\mathcal{F}}_{8}$ presented in Theorem 1.5, and since it is easy to see that this list is closed under the reflection duality operation $(I, \mathfrak{Z}) \mapsto(I, \mathfrak{Z})^{\bullet}$ (2.17), then the Case $2^{\circ}$ applies to $I_{\Gamma}^{*+}$ and therefore the category $I_{\Gamma}^{*+}$-spr is of tame representation type. It follows from Proposition 2.20 (d) that the category $\left(I_{\Lambda}^{*+}, \mathfrak{Z}_{\Lambda} \bullet\right)$-spr is of tame representation type and according to Theorem 3.4 the $D$-order $\Lambda^{\bullet}$ is of tame lattice type, and (a) follows.

Consequently we have proved that the statements (a), (b) and (d) of Theorem 1.5 are equivalent.

The proof of the equivalence $(\mathrm{c}) \Leftrightarrow(\mathrm{d})$ is divided into two parts.

Case $1^{\circ} \cdot n_{3}=0$. From the construction $\Lambda^{\bullet} \mapsto\left(I_{\Lambda}^{*+}, \mathfrak{Z}_{\Lambda} \bullet\right)$ in (3.3) the following three statements are easily derived:

- The sets $C$ and $C^{\prime}$ in the definition of $I_{\Lambda}^{*+}$ are empty, the set $\mathfrak{Z}_{\Lambda} \bullet$ of zerorelations is empty, and $\left(I_{\Lambda}^{*+} \bullet, \mathfrak{Z}_{\Lambda} \bullet\right)$ does not contain the following two-peak posets with zero-relations: $\widehat{\mathcal{F}}_{0}^{1}, \widehat{\mathcal{F}}_{1}^{1}, \widehat{\mathcal{F}}_{1}^{2}, \widehat{\mathcal{F}}_{2}, \widehat{\mathcal{F}}_{3}^{1}, \widehat{\mathcal{F}}_{3}^{2}, \widehat{\mathcal{F}}_{4}, \widehat{\mathcal{F}}_{5}, \widehat{\mathcal{F}}_{6}, \widehat{\mathcal{F}}_{7}$ and $\widehat{\mathcal{F}}_{8}$ presented in Theorem 1.5.

- If $\Lambda_{1}=\Delta_{0}$, then $\left(I_{\Lambda}^{*+}, \mathfrak{Z}_{\Lambda} \bullet\right)=\widehat{\mathcal{F}}_{0}^{3}$. If $\Lambda_{1}$ is one of the $D$-orders $\Delta_{1}, \Delta_{2}, \Delta_{3}$, then $\left(I_{\Lambda}^{*+}, \mathfrak{Z}_{\Lambda} \bullet\right) \cong \widehat{\mathcal{F}}_{0}^{2}$.

- The $D$-order $\Lambda_{1}$ in (1.2) does not contain minor $D$-suborders of the form $\Delta_{0}$ if and only if the two-peak poset $I_{\Lambda}^{*+}$ does not contain as a two-peak subposet the two-peak poset $\widehat{\mathcal{F}}_{0}^{3}$ presented in Theorem 1.5.

- The $D$-order $\Lambda_{1}$ contains a minor $D$-suborder of one of the forms $\Delta_{1}, \Delta_{2}$, $\Delta_{3}$ if and only if the two-peak poset $I_{\Lambda}^{*+}$ contains as a two-peak subposet the two-peak poset $\widehat{\mathcal{F}}_{0}^{2}$ presented in Theorem 1.5.

Hence the equivalence (c) $\Leftrightarrow$ (d) easily follows in case $n_{3}=0$.

$\underline{\text { Case } 2^{\circ}} \cdot n_{3} \geq 1$. First we note that the following four statements are equivalent:

(i) The $D$-order $\Lambda_{1}$ in (1.2) is hereditary of the form (1.6).

(ii) The poset $I^{\prime}=I_{\Lambda_{1}}$ is linearly ordered.

(iii) The poset $\left(I_{\Lambda}^{*+}, \mathfrak{Z}_{\Lambda} \bullet\right) \cong J_{\boldsymbol{\rho}}^{*+}$ (see 3.10$)$ with zero-relations does not contain the poset

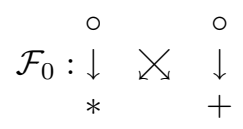

as a two-peak subposet with zero-relations.

(iv) The poset $\left(I_{\Lambda}^{*+}, \mathfrak{Z}_{\Lambda} \bullet\right)$ with zero-relations does not contain any of the posets $\widehat{\mathcal{F}}_{0}^{1}$, $\widehat{\mathcal{F}}_{0}^{2}, \widehat{\mathcal{F}}_{0}^{3}$ presented in Theorem 1.5 as a two-peak subposet with zero-relations.

The implications (i) $\Leftrightarrow($ ii) $\Leftrightarrow$ (iii) $\Rightarrow$ (iv) are immediate consequence of the construction $\Lambda^{\bullet} \mapsto\left(I_{\Lambda}^{*+}, \mathfrak{Z}_{\Lambda} \bullet\right)$ in $(3.3)$.

In order to prove (iv) $\Rightarrow\left(\right.$ iii), assume to the contrary that $\left(I_{\Lambda}^{*+}, \mathfrak{Z}_{\Lambda} \bullet\right)$ contains the two-peak poset $\mathcal{F}_{0}$. Since $n_{3} \geq 1$, each of the chains $C$ and $C^{\prime}$ in the definition of $\left(I_{\Lambda}^{*+}, \mathfrak{Z}_{\Lambda} \bullet\right)(3.3)$ is not empty. Further, since according to our assumption in the theorem the part $\mathcal{X}$ or the part $\mathcal{Y}$ of $\Lambda$ in (1.2) consists of matrices with coefficients 
in $\mathfrak{p}$, then $C$ or $C^{\prime}$ is incomparable with all elements of the subposet $I^{\prime} \equiv I^{\prime \prime}$ of $\left(I_{\Lambda}^{*+}, \mathfrak{Z}_{\Lambda} \bullet\right)$. Since $\mathcal{F}_{0}$ is a two-peak subposet of $\left(I_{\Lambda}^{*+}, \mathfrak{Z}_{\Lambda} \bullet\right)$, then its extension by a point of $C$ or a point of $C^{\prime}$ is a two-peak subposet of $\left(I_{\Lambda}^{*+}, \mathfrak{Z}_{\Lambda} \bullet\right)$ isomorphic with the poset $\mathcal{F}_{0}^{1}$, contrary to our assumption in (iv).

Consequently the conditions (i)-(iv) are equivalent, and therefore in order to finish the proof of $(\mathrm{c}) \Leftrightarrow(\mathrm{d})$ in the case $n_{3} \geq 1$ it remains to show that, in case the $D$-order $\Lambda_{1}$ in (1.2) is hereditary of the form (1.6), the following two conditions are equivalent:

$\left(\mathrm{c}^{\prime}\right)$ The three-partite subamalgam $D$-orders $\Lambda^{\bullet}$ and $\operatorname{rt}(\Lambda)^{\bullet}(1.7)$ do not contain three-partite minor $D$-suborders dominated by any of the 17 three-partite subamalgam $D$-orders listed in the tables of Section $1 \mathrm{~A}$.

$\left(\mathrm{d}^{\prime}\right)$ The two-peak poset $\left(I_{\Lambda^{\bullet}}^{*+}, \mathfrak{Z}_{\Lambda^{\bullet}}\right)$ with zero-relations associated with $\Lambda^{\bullet}$ in $(3.3)$ does not contain as a two-peak subposet with zero-relations any of the following ten hypercritical posets with zero-relations: $\widehat{\mathcal{F}}_{1}^{1}, \widehat{\mathcal{F}}_{1}^{2}, \widehat{\mathcal{F}}_{2}, \widehat{\mathcal{F}}_{3}^{1}, \widehat{\mathcal{F}}_{3}^{2}, \widehat{\mathcal{F}}_{4}, \widehat{\mathcal{F}}_{5}, \widehat{\mathcal{F}}_{6}, \widehat{\mathcal{F}}_{7}$ and $\widehat{\mathcal{F}}_{8}$ presented in Theorem 1.5.

Assume that the $D$-order $\Lambda_{1}$ in (1.2) is hereditary of the form (1.6). For the proof of $\left(\mathrm{d}^{\prime}\right) \Rightarrow\left(\mathrm{c}^{\prime}\right)$ we note first that the two-peak poset with zero-relations $\left(I_{\Lambda^{\bullet}}^{*}, \mathfrak{Z}_{\Lambda^{\bullet}}\right)$ associated with $\Lambda^{\bullet}$ in (3.3) contains as a two-peak subposet with zero-relations any of the hypercritical posets with zero-relations $\widehat{\mathcal{F}}_{1}^{1}, \widehat{\mathcal{F}}_{1}^{2}, \widehat{\mathcal{F}}_{2}, \widehat{\mathcal{F}}_{3}^{1}, \widehat{\mathcal{F}}_{3}^{2}, \widehat{\mathcal{F}}_{4}, \widehat{\mathcal{F}}_{5}, \widehat{\mathcal{F}}_{6}, \widehat{\mathcal{F}}_{7}$, $\widehat{\mathcal{F}}_{8}$ presented in Theorem 1.5 if $\Lambda^{\bullet}$ is one of the $D$-orders $\Omega_{1}^{\bullet}, \ldots, \Omega_{17}^{\bullet}$ presented in the tables of Section 1A. More precisely, if $\Omega_{j}^{\bullet}$ is of type $\widehat{\mathcal{F}}_{j}$ in the notation of Section 1 , then $\left(I_{\Omega_{j}^{\bullet}}^{*+}, \mathfrak{Z}_{\Omega_{j}^{\bullet}}\right)$ contains $\widehat{\mathcal{F}}_{j}$. For example, $\left(I_{\Omega_{1}^{*}}^{*+}, \mathfrak{Z}_{\Omega_{1}^{\bullet}}\right)=\widehat{\mathcal{F}}_{1}^{1}$. The poset with zero-relations $\left(I_{\Omega_{2}^{*}}^{*+}, \mathfrak{Z}_{\Omega_{2}^{\bullet}}\right)$ has the form

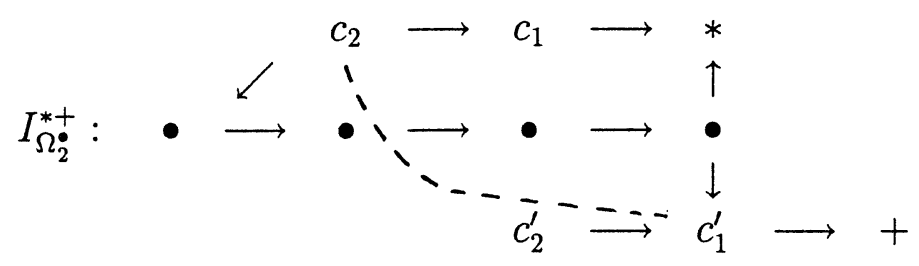

and $\mathfrak{Z}_{\Omega_{2}^{\bullet}}=\left\{\left(c_{2}, c_{1}^{\prime}\right),\left(c_{2},+\right)\right\}$. It follows that the poset with zero-relations $\left(I_{\Omega_{2}^{*}}^{*+}, \mathfrak{Z}_{\Omega_{2}^{\bullet}}\right)$ contains the poset $\widehat{\mathcal{F}}_{1}^{1}$ as the subposet with zero-relations obtained by omitting the points $c_{2}$ and $c_{1}^{\prime}$. The proof in the remaining cases is left to the reader.

It follows from Theorem 3.4 (iii) that the $D$-orders $\Omega_{1}^{\bullet}, \ldots, \Omega_{17}^{\bullet}$ are of wild lattice type, because in view of the reflection duality (2.19), Proposition 2.20 (d) and [16, Theorem 1.3] the category $\left(I_{\Omega_{j}^{*}}^{*+}, \mathfrak{Z}_{\Omega_{j}^{\bullet}}\right)$-spr is of wild representation type for $j=1, \ldots, 17$.

In order to prove $\left(\mathrm{d}^{\prime}\right) \Rightarrow\left(\mathrm{c}^{\prime}\right)$, assume to the contrary that the three-partite $D$ order $\Lambda^{\bullet}$ contains a three-partite minor $D$-suborder $\Gamma^{\bullet}=e \Lambda^{\bullet} e$, where $e \in \Lambda^{\bullet}$ is an idempotent, and $\Gamma^{\bullet}$ is dominated by $\Omega^{\bullet} \cong \Omega_{j}^{\bullet}$ for some $j$. Then $\Omega^{\bullet}$ is of wild lattice type, and according to Lemmas 4.3 and 4.4 the order $\Lambda^{\bullet}$ is also of wild lattice type. By the tame-wild dichotomy and the equivalences $(\mathrm{a}) \Leftrightarrow(\mathrm{d}) \Leftrightarrow\left(\mathrm{d}^{\prime}\right)$ proved above, $\left(I_{\Lambda^{\bullet}}^{*+}, \mathfrak{Z}_{\Lambda} \bullet\right)$ contains any of the hypercritical posets with zero-relations $\widehat{\mathcal{F}}_{1}^{1}, \widehat{\mathcal{F}}_{1}^{2}, \widehat{\mathcal{F}}_{2}$, $\widehat{\mathcal{F}}_{3}^{1}, \widehat{\mathcal{F}}_{3}^{2}, \widehat{\mathcal{F}}_{4}, \widehat{\mathcal{F}}_{5}, \widehat{\mathcal{F}}_{6}, \widehat{\mathcal{F}}_{7}, \widehat{\mathcal{F}}_{8}$, contrary to our assumption $\left(\mathrm{d}^{\prime}\right)$.

Let us give an alternative and direct proof of the above fact. Since $\Gamma^{\bullet}$ is a threepartite minor suborder of $\Lambda^{\bullet}$, then $\left(I_{\Lambda^{\bullet}}^{*+}, \mathfrak{Z}_{\Lambda^{\bullet}}\right)$ contains $\left(I_{\Gamma^{\bullet}}^{*+}, \mathfrak{Z}_{\Gamma^{\bullet}}\right)$. Since $\Omega^{\bullet}=\Omega_{j}^{\bullet}$ 
dominates $\Gamma^{\bullet}$, then $\left(I_{\Omega^{\bullet}}^{*+}, \mathfrak{Z}_{\Omega^{\bullet}}\right)$ is obtained from $\left(I_{\Gamma^{\bullet}}^{*+}, \mathfrak{Z}_{\Gamma^{\bullet}}\right)$ by adding new relations of the forms $c \prec i$ and $j \prec c^{\prime}$, where $i, j \in I^{\prime} \equiv I^{\prime \prime}, c \in C$ and $c^{\prime} \in C^{\prime}$. Note that $\left(I_{\Omega_{j}^{+}}^{*+}, \mathfrak{Z}_{\Omega_{j}^{\bullet}}\right)$ has no relation of the above form for $j \notin\{4,5\}$. It follows that in this case $\left(I_{\Omega_{j}^{+}}^{*+}, \mathfrak{Z}_{\Omega_{j}^{\bullet}}\right)$ is contained in $\left(I_{\Lambda^{\bullet}}^{*+}, \mathfrak{Z}_{\Lambda} \bullet\right)$, contrary to our assumption. If $j=4$ or $j=5$, a simple analysis shows that either $\left(I_{\Lambda^{\bullet}}^{*+}, \mathfrak{Z}_{\Lambda} \bullet\right)$ contains $\left(I_{\Omega_{j}^{*}}^{*+}, \mathfrak{Z}_{\Omega_{j}^{\bullet}}\right)$, or else $\left(I_{\Lambda}^{*+}, \mathfrak{Z}_{\Lambda} \bullet\right)$ contains the poset $\widehat{\mathcal{F}}_{1}^{1}$, contrary to our assumption. This finishes the proof of the implication $\left(\mathrm{d}^{\prime}\right) \Rightarrow\left(\mathrm{c}^{\prime}\right)$.

The proof of the implication $\left(\mathrm{c}^{\prime}\right) \Rightarrow\left(\mathrm{d}^{\prime}\right)$ reduces to pure combinatorial poset properties by applying the constructions

$$
\Lambda^{\bullet} \mapsto I_{\Lambda} \bullet, \sigma \mapsto\left(I_{\Lambda}^{*+}, \mathfrak{Z}_{\Lambda} \bullet\right),
$$

where $I_{\Lambda} \bullet, \sigma=\left(I_{\Lambda}, \preceq, I^{\prime}, C, I^{\prime \prime}, \sigma: I^{\prime} \rightarrow I^{\prime \prime}\right)$ is the bipartite stratified poset (3.2) and $\left(I_{\Lambda}^{*+}, \mathfrak{Z}_{\Lambda} \bullet\right)$ is the two-peak poset with zero-relations (3.3).

The following properties of the constructions follow directly from the definitions.

(A) The $D$-order $\Lambda$ together with its three-partition shown in (1.2) is uniquely determined by the bipartite stratified poset $I_{\Lambda} \bullet, \sigma$. Hence the three-partite subamalgam $D$-order $\Lambda^{\bullet}(1.3)$ is uniquely determined by $I_{\Lambda} \bullet, \sigma$.

(B) A three-partite subamalgam $D$-order $\Gamma^{\bullet}$ is a three-partite minor $D$-suborder of $\Lambda^{\bullet}$ if and only if $I_{\Gamma}, \tau$ is a bipartite stratified subposet of $I_{\Lambda} \bullet, \sigma$.

(C) For any bipartite stratified subposet $J_{\tau}=\left(J, \preceq, J^{\prime}, C, J^{\prime \prime}, \tau: J^{\prime} \rightarrow J^{\prime \prime}\right)$ of $I_{\Lambda} \bullet, \sigma$ there exists a unique three-partite minor $D$-suborder $\Gamma$ of $\Lambda$ such that $I_{\Gamma} \cdot \tau=J_{\tau}$.

(D) A three-partite $D$-order $\Lambda^{\prime}$ of the form (1.2) dominates a three-partite $D$ order $\Lambda$ if and only if $I^{\prime}=I_{\Lambda_{1}}=I_{\Lambda_{1}^{\prime}}, I^{\prime \prime}=I_{\Lambda_{2}}=I_{\Lambda_{2}^{\prime}}, C=I_{\Lambda_{3}}=I_{\Lambda_{3}^{\prime}}$ (a poset equality) and the partial order relation of $I_{\Lambda^{\prime}}$ is obtained from the partial order relation of $I_{\Lambda}$ by adding finitely many new relations $i^{\prime} \preceq c_{1}, c_{2} \preceq i^{\prime \prime}$, where $i^{\prime} \in I^{\prime}$, $i^{\prime \prime} \in I^{\prime \prime}$ and $c_{1}, c_{2} \in C$.

(E) If the two-peak poset with zero-relations $\left(I_{\Lambda}^{*+}, \mathfrak{Z}_{\Lambda} \bullet\right)$ is given, then the poset $I^{\prime} \equiv I^{\prime \prime}$ can be reconstructed as the subposet of $I_{\Lambda}^{*+}$ consisting of all points $s$ such that $s \preceq *, s \preceq+$ and each of the pairs $(s, *)$ and $(s,+)$ does not belong to the set $\mathfrak{Z}_{\Lambda} \bullet$ of zero-relations. Moreover, $C \cup C^{\prime}=I_{\Lambda}^{*+} \backslash\left(I^{\prime} \equiv I^{\prime \prime}\right)$ in the notation of (3.3).

It follows that the classification of minimal three-partite subamalgam $D$-orders $\Lambda^{\bullet}$ of wild lattice type can be given by means of bipartite stratified subposets of $I_{\Lambda} \bullet, \sigma \cdot$

In this way we shall show that if $\Lambda$ is a three-partite $D$-order (1.2) and the associated two-peak poset with zero-relations $\left(I_{\Lambda}^{*+}, \mathfrak{Z}_{\Lambda} \bullet\right)$ contains one of the hypercritical posets with zero-relations $\widehat{\mathcal{F}}_{1}^{1}, \widehat{\mathcal{F}}_{1}^{2}, \widehat{\mathcal{F}}_{2}, \widehat{\mathcal{F}}_{3}^{1}, \widehat{\mathcal{F}}_{3}^{2}, \widehat{\mathcal{F}}_{4}, \widehat{\mathcal{F}}_{5}, \widehat{\mathcal{F}}_{6}, \widehat{\mathcal{F}}_{7}, \widehat{\mathcal{F}}_{8}$ as a two-peak subposet with zero-relations, then the subamalgam $D$-order $\Lambda^{\bullet}(1.3)$ contains a three-partite minor $D$-suborder $\Gamma^{\bullet}$ which is dominated by any of the $D$-orders $\Omega_{1}^{\bullet}, \ldots, \Omega_{17}^{\bullet}$ shown in the tables of Section $1 \mathrm{~A}$.

For example we assume that $\Lambda$ is a three-partite $D$-order of the form (1.2) such that $\left(I_{\Lambda}^{*+}, \mathfrak{Z}_{\Lambda} \bullet\right)$ contains the poset

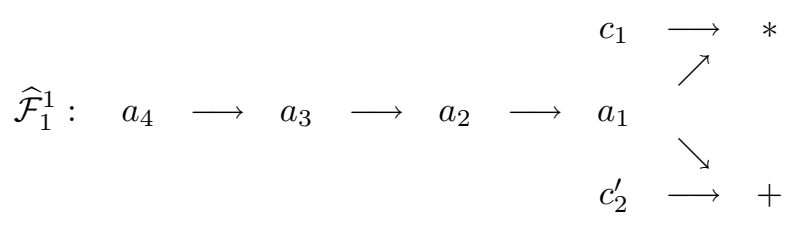


and $\left(I_{\Lambda^{\bullet}}^{*+}, \mathfrak{Z}_{\Lambda^{\bullet}}\right)$ does not contain the poset $\widehat{\mathcal{F}}_{2}$. We shall show that the subamalgam $D$-order $\Lambda^{\bullet}$ contains a three-partite minor $D$-suborder $\Omega^{\bullet}$ which is dominated by the $D$-order $\Omega_{1}^{\bullet}$ or by $\Omega_{2}^{\bullet}$ shown in Section $1 \mathrm{~A}$.

Look at the bipartite stratified poset $I_{\Lambda} \bullet, \sigma=\left(I_{\Lambda}, \preceq, I^{\prime}, C, I^{\prime \prime}, \sigma: I^{\prime} \rightarrow I^{\prime \prime}\right)(3.2)$. Recall that $C$ is a chain, the elements $c_{1}, c_{2}$ belong to $C$, and $c_{2}^{\prime}$ denotes a copy of $c_{2}$ in $C^{\prime} \subseteq I_{\Lambda}^{*+}$ (see (3.3)). Without loss of generality we may suppose that $a_{4} \preceq a_{3} \preceq a_{2} \preceq a_{1}$ is a chain in $I^{\prime}$ and $a_{4}^{\prime} \preceq a_{3}^{\prime} \preceq a_{2}^{\prime} \preceq a_{1}^{\prime}$ is the image of $a_{4} \preceq a_{3} \preceq a_{2} \preceq a_{1}$ under the poset isomorphism $\sigma: I^{\prime} \rightarrow I^{\prime \prime}$. It follows from our assumption on the bipartition (1.2) that $a_{1} \preceq a_{4}^{\prime}$.

Let $\Gamma$ be a three-partite minor of $\Lambda$ (1.2) defined by the rows and columns numbered by the elements $a_{4}, a_{3}, a_{2}, a_{1}, a_{4}^{\prime}, a_{3}^{\prime}, a_{2}^{\prime}, a_{1}^{\prime}, c_{1}, c_{2}$. By our assumption

$$
I_{\Gamma \bullet, \sigma}=\left(J_{\Gamma}, \preceq, J^{\prime}, \bar{C}, J^{\prime \prime}, \sigma: J^{\prime} \rightarrow J^{\prime \prime}\right),
$$

where $J^{\prime}=\left\{a_{4} \preceq a_{3} \preceq a_{2} \preceq a_{1}\right\} \subset I^{\prime}, J^{\prime \prime}=\left\{a_{4}^{\prime} \preceq a_{3}^{\prime} \preceq a_{2}^{\prime} \preceq a_{1}^{\prime}\right\} \subset I^{\prime \prime}$, $\bar{C}=\left\{c_{1}, c_{2}\right\} \subseteq C$, and $\sigma: J^{\prime} \rightarrow J^{\prime \prime}$ is given by $\sigma\left(a_{1}\right)=a_{1}^{\prime}, \sigma\left(a_{2}\right)=a_{2}^{\prime}, \sigma\left(a_{3}\right)=a_{3}^{\prime}$ and $\sigma\left(a_{4}\right)=a_{4}^{\prime}$.

It follows from the shape of $\widehat{\mathcal{F}}_{1}^{1}$ that $c_{1}$ is not comparable with the chain $a_{4}^{\prime} \rightarrow$ $a_{3}^{\prime} \rightarrow a_{2}^{\prime} \rightarrow a_{1}^{\prime}$ in the poset $I_{\Lambda}$ and $c_{2}$ is not comparable with the chain $a_{4} \rightarrow a_{3} \rightarrow$ $a_{2} \rightarrow a_{1}$, and either $c_{1}=c_{2}$ or else $c_{2} \prec c_{1}$.

In case $c_{1}=c_{2}$ we conclude from $(\mathbf{A})-(\mathbf{C})$ and from the shape of the bipartite stratified poset $I_{\Gamma \cdot \sigma}$ that $\Gamma=\Omega_{1}$.

Now consider the case $c_{2} \prec c_{1}$. Since $\left(I_{\Gamma^{\bullet}}^{*+}, \mathfrak{Z}_{\Gamma} \bullet\right)$ does not contains the poset $\widehat{\mathcal{F}}_{2}$, it follows from the above observations and $(\mathbf{A})-(\mathbf{E})$ that the poset $J_{\Gamma}=J^{\prime} \cup \bar{C} \cup J^{\prime \prime}$ has the following structure:

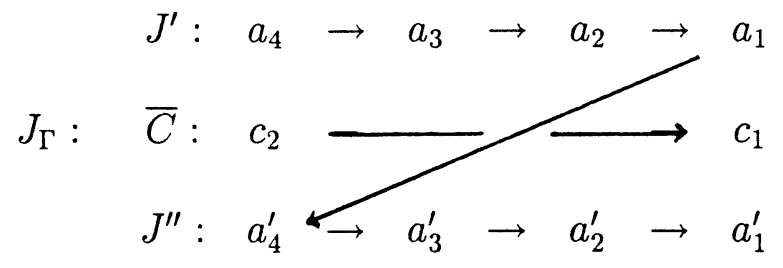

with some relations from $J^{\prime}$ to $\bar{C}$ and from $\bar{C}$ to $J^{\prime \prime}$. It follows from (D) that in his class any $D$-order $\Gamma$ is dominated by a unique three-partite $D$-order $\Omega$ corresponding to the bipartite stratified poset

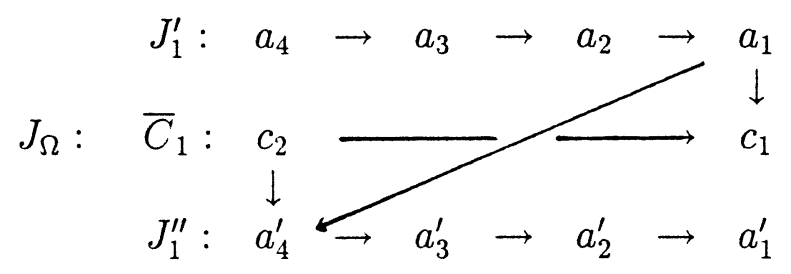

(see the proof of the implication $(\mathrm{d}) \Rightarrow(\mathrm{c})$ in [40, Section 5]). It is clear that $\Omega$ is just the $D$-order $\Omega_{2}$ in the tables of Section $1 \mathrm{~A}$.

It follows from the above analysis that, up to domination and minors, the minimal three-partite $D$-orders $(1.2)$ such that $\left(I_{\Lambda}^{*+}, \mathfrak{Z}_{\Lambda} \bullet\right)$ contains the poset $\widehat{\mathcal{F}}_{1}^{1}$ and $\left(I_{\Lambda^{\bullet}}^{*+}, \mathfrak{Z}_{\Lambda} \bullet\right)$ does not contain the poset $\widehat{\mathcal{F}}_{2}$ are just the $D$-orders $\Omega_{1}$ and $\Omega_{2}$ shown in Section $1 \mathrm{~A}$. 
By the technique applied above we also prove that if $\left(I_{\Lambda}^{*+} \bullet, \mathfrak{Z}_{\Lambda} \bullet\right)$ contains any of the hypercritical posets with zero-relations $\widehat{\mathcal{F}}_{1}^{1}, \widehat{\mathcal{F}}_{1}^{2}, \widehat{\mathcal{F}}_{2}, \widehat{\mathcal{F}}_{3}^{1}, \widehat{\mathcal{F}}_{3}^{2}, \widehat{\mathcal{F}}_{4}, \widehat{\mathcal{F}}_{5}, \widehat{\mathcal{F}}_{6}, \widehat{\mathcal{F}}_{7}, \widehat{\mathcal{F}}_{8}$ (see Theorem 1.5) as a two-peak subposet with zero-relations, then the three-partite order $\Lambda^{\bullet}$ contains a three-partite minor $D$-suborder $\Gamma^{\bullet}$ dominated by a $D$-order $\Omega^{\bullet}$ of one of the 17 forms shown in the tables of Section 1A. The details are left to the reader. This completes the proof of Theorem 1.5.

Question 4.7. Does Theorem 1.5 remain valid if we remove the assumption that the part $\mathcal{X}$ or the part $\mathcal{Y}$ of the $D$-order $\Lambda$ in (1.2) consists of matrices with coefficients in $\mathfrak{p}$ ?

\section{REFERENCES}

[1] D. M. Arnold, Representations of partially ordered sets and abelian groups, Contemporary Math. 87 (1989), 91-109. MR 90j:20118

[2] D. M. Arnold and M. Dugas, Block rigid almost completely decomposable groups and lattices over multiple pullback rings, J. Pure Appl. Alg. 87 (1993), 105-121. [MR 94f:20102]

[3] D. M. Arnold, F. Richman and C. Vinsonhaler, Representations of finite posets and valuated groups, J. Algebra 155 (1993), 110-126. MR 94d:20064

[4] K. Bongartz, A criterion for finite representation type, Math. Ann. 269 (1984), 1-12. MR 86k:16023

[5] Ch. W. Curtis and I. Reiner, "Methods of Representation Theory", Vol. I,, Wiley Classics Library Edition, New York, 1990. MR 90k:20001

[6] P. Dowbor and S. Kasjan, Galois covering technique and tame non-simply connected posets of polynomial growth, J. Pure Appl. Algebra 147 (2000), 1-24.

[7] P. Dowbor and A. Skowroński, On Galois coverings of tame algebras, Arch. Math. 44 (1985), 522-529. MR 87a:16035

[8] P. Dowbor and A. Skowroński, Galois coverings of representation-infinite algebras, Comment. Math. Helv. 62 (1987), 311-337. MR 88m:16020

[9] J. A. Drozd and M. G. Greuel, Tame-wild dichotomy for Cohen-Macaulay modules, Math. Ann. 294 (1992), 387-394. MR 93h:16023

[10] Y. A. Drozd, Cohen-Macaulay modules and vector bundles, Proc. Euroconference "Interactions between Ring Theory and Representations of Algebras", Murcia, 12-17 January 1998, Lecture Notes in Pure and Appl. Math. (to appear).

[11] E. L. Green and I. Reiner, Integral representations and diagrams, Michigan Math. J. 25 (1978), 53-84. MR 80g:16032

[12] J. Haefner and L. Klingler, Special quasi-triads and integral group rings of finite representation type, I and II, J. Algebra 158 (1993), 279-374. MR 94k:16028

[13] H.-J. von Höhne and D. Simson, Bipartite posets of finite prinjective type, J. Algebra 201 (1998), 86-114. MR 99h:16027

[14] S. Kasjan, Minimal bipartite algebras of infinite prinjective type with prin-preprojective component, Colloquium Math. 76 (1998), 295-317. MR 99b:16024

[15] S. Kasjan and D. Simson, Varieties of poset representations and minimal posets of wild prinjective type, in Proceedings of the Sixth International Conference on Representations of Algebras, vol. 14, Canadian Mathematical Society Conference Proceedings, 1993, pp. 245284. MR 94e: 16025

[16] S. Kasjan and D. Simson, Fully wild prinjective type of posets and their quadratic forms, $J$. Algebra 172 (1995), 506-529. MR 96m:16020

[17] S. Kasjan and D. Simson, Tame prinjective type and Tits form of two-peak posets I, J. Pure Appl. Algebra 106 (1996), 307-330. MR 97d:16018

[18] S. Kasjan and D. Simson, Tame prinjective type and Tits form of two-peak posets II, J. Algebra 187 (1997), 71-96. MR 98h:16020

[19] S. Kasjan and D. Simson, A subbimodule reduction, a peak reduction functor and tame prinjective type, Bull. Pol. Acad. Sci. Math. 45 (1997), 89-107. MR 99a:16009]

[20] J. Kosakowska and D. Simson, On Tits form and prinjective representations of posets of finite prinjective type, Comm. Algebra 26 (1998), 1613-1623. MR 99d:16013 
[21] L. A. Nazarova, Partially ordered sets of infinite type, Izv. Akad. Nauk SSSR 39 (1975), 963-991; English transl., Math. USSR Izv. 9 (1975), 911-938. MR 55:10604

[22] L. A. Nazarova and V. A. Roiter, Representations of bipartite completed posets, Comment. Math. Helv. 63 (1988), 498-526. MR 89m:06003]

[23] J. A. de la Peña and D. Simson, Prinjective modules, reflection functors, quadratic forms and Auslander-Reiten sequences, Trans. Amer. Math. Soc. 329 (1992), 733-753. MR 92e:16005

[24] I. Reiner, "Maximal Orders", Academic Press, London, 1975. MR 52:13910

[25] K. W. Roggenkamp, Auslander-Reiten species of Bäckstrom orders, J. Algebra 85 (1983), 449-476. MR 85g:16005

[26] K. W. Roggenkamp, Lattices over subhereditary orders and socle-projective modules, J. Algebra 121 (1989), 40-67. MR 90h:16016

[27] C. M. Ringel and K. W. Roggenkamp, Diagrammatic methods in the representation theory of orders, J. Algebra 60 (1979), 11-42. MR 81b:16008

[28] D. Simson, Socle reductions and socle projective modules, J. Algebra 103 (1986), 18-68. MR 88a:16058

[29] D. Simson, Representations of bounded stratified posets, coverings and socle projective modules, in "Topics in Algebra, Part I: Rings and Representations of Algebras", Banach Center Publications, vol. 26, PWN, Warszawa, 1990, pp. 499-533. MR 93g:16019

[30] D. Simson, A splitting theorem for multipeak path algebras, Fund. Math. 138 (1991), 113137. MR 93a:16007

[31] D. Simson, Right peak algebras of two-separate stratified posets, their Galois coverings and socle projective modules, Comm. Algebra 20 (1992), 3541-3591. MR 94f:16031

[32] D. Simson, "Linear Representations of Partially Ordered Sets and Vector Space Categories", Algebra, Logic and Applications, Vol. 4, Gordon \& Breach Science Publishers, New York, 1992. MR 95g:16013

[33] D. Simson, Posets of finite prinjective type and a class of orders, J. Pure Appl. Algebra 90 (1993), 77-103. MR 95b:16011

[34] D. Simson, On representation types of module subcategories and orders, Bull. Pol. Acad. Sci., Math. 41 (1993), 77-93. MR 97g:16024

[35] D. Simson, A reduction functor, tameness and Tits form for a class of orders, J. Algebra 174 (1995), 430-452. MR 96d:16022

[36] D. Simson, Triangles of modules and non-polynomial growth, C. R. Acad. Sci. Paris, Série I 321 (1995), 33-38. MR 96g:16015

[37] D. Simson, Representation embedding problems, categories of extensions and prinjective modules, in Proceedings of the Seventh International Conference on Representations of Algebras, Canadian Mathematical Society Conference Proceedings, Vol. 18, 1996, 601-639. MR 98g:16011

[38] D. Simson, Socle projective representations of partially ordered sets and Tits quadratic forms with application to lattices over orders, in Proceedings of the Conference on Abelian Groups and Modules, Colorado Springs, August 1995, Lecture Notes in Pure and Appl. Math., Vol. 182, 1996, pp. 73-111. MR 97j:16024

[39] D. Simson, Prinjective modules, propartite modules, representations of bocses and lattices over orders, J. Math. Soc. Japan 49 (1997), 31-68. MR 98e:16015

[40] D. Simson, Three-partite subamalgams of tiled orders of finite lattice type, J. Pure Appl. Algebra 138 (1999), 151-184. CMP 99:12

[41] D. Simson, Representation types, Tits reduced quadratic forms and orbit problems for lattices over orders, in Proc. AMS-IMS-SIAM Summer Research Conference "Trends in the Representation Theory of Finite Dimensional Algebras", The University of Washington, July 20-24, 1997, Contemporary Math. 229 (1998), 307-342. CMP 99:09

[42] D. Simson, Tame three-partite subamalgams of tiled orders of polynomial growth, Colloq. Math. 81 (1999), 237-262.

[43] D. Simson, Cohen-Macaulay modules over classical orders, Proc. Euroconference "Interactions between Ring Theory and Representations of Algebras", Murcia, 12-17 January 1998, Lecture Notes in Pure and Appl. Math. (to appear).

[44] Y. Yoshino, "Cohen-Macaulay Modules over Cohen-Macaulay Rings", London Math. Soc. Lecture Notes Series, Vol. 146, Cambridge University Press, 1990. MR 92b:13016 
[45] A. G. Zavadskij and V. V. Kirichenko, Torsion-free modules over prime rings, Zap. Naychn. Sem. Leningrad. Otdel. Mat. Inst. Steklov. (LOMI) 57 (1976), 100-116; English tranl., J. Soviet Math. 11 (1979), 598-612. MR 58:11026

[46] A. G. Zavadskij and V. V. Kirichenko, Semimaximal rings of finite type, Mat. Sbornik 103 (1977), 323-345; English transl., Math. USSR Sb. 32 (1997), 273-291. MR 56:15706

[47] A. G. Zavadskij, Representations of partially ordered sets of finite growth, Kievskij Ordena Trudovovo Krasnovo Znameni Inžinerno-Stroitelnyi Institut (KISI), Dep. Ukr. NIINTI, No. 413-Yk-D83, Kiev, 1983, pp. 1-76 (Russian).

[48] A. G. Zavadskij, Differentiation algorithm and classification of representations, Izv. Akad. Nauk SSSR, Ser. Mat. 55 (1991), 1007-1048 (in Russian); English transl., Math. USSR Izvestia 39 (1992), 975-1012.

Faculty of Mathematics and Informatics, Nicholas Copernicus University, Ul. Chopina 12/18, 87-100 Torún, Poland

E-mail address: simson@mat.uni.torun.pl 